\title{
Zooplankton community composition of high mountain lakes in the Tatra Mts., the Alps in North Tyrol, and Scotland: relationship to $\mathrm{pH}$, depth, organic carbon, and chlorophyll-a concentration
}

Ivan Skála

\begin{abstract}
Zooplankton community composition of high mountain lakes in the Tatra Mts., the Alps in North Tyrol, and Scotland: relationship to $\mathrm{pH}$, depth, organic carbon, and chlorophyll-a concentration. Acta Mus. Siles. Sci. Natur., 64: 175-189, 2015.

Abstract: The European EMERGE (European Mountain lake Ecosystems: Regionalisation, diaGnostic \& socio-economic Evaluation) project was a survey of high mountain lakes (above treeline) across Europe using unified methods of sampling and analysis. The sampling was carried out in summer or autumn 2000, and comprised biological samples, and samples for chemical analysis. Data from three lake districts are used in this paper: the Tatra Mts. in Slovakia and Poland (45 lakes), the Alps in Tyrol in Austria (22 lakes), and Scotland (30 lakes). As it is shown by multiple regression analysis, DTOC (dissolved or total organic carbon) is the key variable for most groups of zooplankton. With increasing DTOC and mostly with chlorophyll-a decreasing, $\mathrm{pH}$ increasing and depth decreasing, macrofitrators with coarse filter meshes are replaced by microfiltrators with fine filter meshes. Higher DTOC may increase bacterioplankton production and advantage species able to consume bacteria (microfiltrators). Other zooplankton species also differ in their preference for DTOC, chlorophyll-a, $\mathrm{pH}$ and depth, but DTOC being positively correlated with chlorophyll-a and $\mathrm{pH}$ positively correlated with depth. It may be caused by their different preference for food quality in terms of C:P ratio.
\end{abstract}

Key words: zooplankton, alpine, mountain, dissolved organic carbon, food quality

\section{Introduction}

It is known that zooplankton species differ in the ratio of algae and bacteria in their food (Geller \& Müller 1981; Hessen 1985; Hessen et al. 1989). Their ability to collect bacteria is dependent on the density of their filter meshes (Brendelberger 1991).

Bacteria may be an important source of phosphorus for zooplankton, because they have a higher phosphorus content than algae. For example, Vadstein et al. (1993) found 10 times lower C:P ratio in bacteria than in algae in Lake Nesjøvatn in Norway. Hessen and Andersen (1990) found bacteria to account for $75 \%$ of particulate phosphorus in a humic lake, which made them the most important source of phosphorus for zooplankton. Moreover, assimilation efficiency of phosphorus from bacteria-sized particles was almost 82\% (Hessen \& Andersen 1990).

At a low ambient phosphorus concentration and elevated carbon content, C:P ratio of phytoplankton is so high that it limits the growth rate of zooplankton consuming it (DeMott et al. 1998; Elser et al. 2001; Hessen et al. 2002; Urabe et al. 2002). At high C:P, the gross (growth to ingestion ratio) and net (growth to assimilation ratio) growth efficiencies of Daphnia for phosphorus decrease, so that the phosphorus cannot be used for growth, and the growth rate decreases (DeMott et al. 1998). Copepods and carnivorous Cladocera have a lower P content in comparison with herbivorous Cladocera (Andersen \& Hessen 1991; Hessen \& Lyche 1991). These species could be less affected by low P content in food (Urabe et al. 2002). 
Increasing intensity of light increases carbon to phosphorus (C:P) ratio of phytoplankton due to increased carbon fixation which elevates the carbon content (Hessen et al. 2002; Urabe et al. 2002; Hessen 2008). Since light intensity decreases with increasing depth of lakes, lake depth may influence food quality for zooplankton in terms of C:P ratio.

The ratio of algal to bacterial production is dependent on the concentration of the substrate for bacterial growth, which is dissolved organic carbon (DOC). In lakes with low primary production, the primary production to bacterioplankton production ratio gradually decreases with increasing allochthonous DOC concentration, because DOC has a positive influence on bacterial production and a negative influence on primary production due to absorption of light (Jansson et al. 2000). Based on summer epilimnetic data from oligotrophic clearwater lakes and dystrophic humic lakes, the ratio equals 1 at DOC concentrations around $10 \mathrm{mg} \mathrm{dm}^{-3}$ (Jansson et al. 2000). In more productive lakes and the ocean, allochthonous DOC is less important, and bacterial production is positively correlated with algal primary production (Cole et al. 1988). In mountain lakes, Reche et al. (1996) and Panzenböck (2007) found that the amount of carbon released extracellularly by phytoplankton was large enough to satisfy the bacterioplankton demand. The contribution of photosynthetic extracellular release (PER) to primary production was on average $32 \%$ in the alpine lake Gossenköllesee (Panzenböck 2007). The contribution of net PER to total net primary production increased with increasing irradiance. 15 times higher rates of PER were observed during the ice-free season. $37 \%$ of the carbon released during incubation was incorporated into bacterial biomass.

In acidic lakes, extracellular release of DOC by phytoplankton may also be limited by dissolved inorganic carbon concentration (DIC). Low DIC supply may limit the growth and photosynthesis of phytoplankton. At $\mathrm{pH}$ less than 6, the DIC resulting from air equilibration is about $10 \mu \mathrm{M}$, a value known to be rate limiting for various species in culture (Goldman \& Graham 1981; Williams \& Turpin 1987), and for natural phytoplankton (Nygaard 1989; Hein 1997). This level of DIC may be even below the photosynthetic compensation point of some algae species, and preclude their growth (Williams \& Turpin 1987). However, lake waters are sometimes naturally $\mathrm{CO}_{2}$ supersaturated, and then inorganic carbon limitation does not occur (Hein 1997).

It follows from the discussion above that bacteria are an important high quality food source for zooplankton. However, the importance of bacteria as food is determined by the size of bacterial production, which is stimulated by DOC. The in-lake photosynthetic production of DOC by algae is important, but decreases with decreasing irradiance caused by depth and also probably decreases at low $\mathrm{pH}$. High levels of DOC may thus be awaited in lakes with high import of allochthonous DOC, or in lakes with high production DOC, which is probable at high chlorophyll-a concentration, high irradiance in shallow lakes and possibly at high $\mathrm{pH}$. In the lakes with high bacterial production stimulated by high DOC, the ability to collect bacteria should advantage microfiltrators in zooplankton. The aim of this work is to confirm that the distribution of micro- and macrofitrators in high mountain lakes (above the tree-line) is determined by DTOC (dissolved or total organic carbon), chlorophyll-a, $\mathrm{pH}$ and depth in three mountain areas in Europe.

\section{Methods}

High mountain lakes situated above the tree-line were surveyed. Samples were collected in the lakes of three lake districts covered by the EMERGE project: the Tatra Mts. in Slovakia and Poland (45 lakes), the Alps in Tyrol in Austria (22 lakes) and Scotland (30 lakes) (Fig. 1; Table 1). The Scottish sites were located in the Cairngorm and Grampian Mountains and the northwest Highlands (Kernan et al. 2009a). Sampling survey was performed in the ice-free season in late summer or autumn 2000: in the Tatra Mts. in 16.9.-1.10., in the Alps in 16.8.-19.9. and in Scotland in 19.9.-25.10. Sampling and chemical analysis were performed by many colleagues across Europe. The methods of chemical analyses were described by Camarero et al. (2009). Water samples 
were collected as surface samples from central part of each lake (Straškrábová et al. 2009), and kept in cool and dark conditions until analysis. Chemical analyses were carried out by all involved laboratories according to the methods agreed within the MOLAR Project (Camarero et al. 2009), which were described by Wathne \& Hansen (1997), and The MOLAR Water Chemistry Group (1999). Total organic carbon (TOC) was determined using catalytic combustion and IR spectrophotometry detection with TOC5000 Analyser, Shimadzu, Kyoto, Japan (Camarero et al. 2009). Only one depth with expected maximum chlorophyll-a concentration was sampled for bacterial biomass, chlorophyll-a concentration, and phytoplankton (Straškrábová \& Stuchlík 2000). During autumnal mixing, the samples were taken with the top end of a sampler $0.5 \mathrm{~m}$ below the surface (so in Scotland according to Kernan et al. 2009a), whereas in deeper thermally stratified lakes at the depth of 1.5 times Secchi depth reading, which is where the chlorophyll maximum occurs during summer stratification (Catalan et al. 2002). Chlorophyll-a samples underwent filtration and concentration on Whatman GF/ glass fibre filters, and chlorophyll-a was then extracted in acetone and measured spectrophotometrically or fluorometrically (Kernan et al. 2009c; Straškrábová et al. 2009). In Scotland, the concentration of chlorophyll-a was determined spectrophotometrically after hot extraction in a mixture of acetone and methanol 5:1 (Kernan et al. 2009a). Large pelagic zooplankton was sampled quantitatively (quantitative samples) using Apstein type pelagic net (with $200 \mu \mathrm{m}$ mesh and $0.2 \mathrm{~m}$ diameter except $0.1 \mathrm{~m}$ diameter in the Alps) hauled several times from the bottom to the surface at the deepest part of the lake (Kernan et al. 2009b; Kernan et al. 2009c). In Scotland, the net was only hauled from 1.5x Secchi disc depth (Kernan et al. 2009a). All pelagic zooplankton was sampled qualitatively (qualitative samples) using pelagic net (with $40 \mu \mathrm{m}$ mesh and $0.2 \mathrm{~m}$ diameter) hauled vertically and laterally. Zooplankton samples were preserved with formaldehyde at the final concentration of $4 \%$. Planktonic organisms in quantitative samples were counted in an Utermöhl type chamber using an inverted microscope. Sub-sampling was used to count at least 400 individuals of large crustaceans in a known part of each sample. Concentrated qualitative samples were observed spread on microscope slides and the found taxa were recorded. A part of each sample covered by 2-5 cover slips was observed.

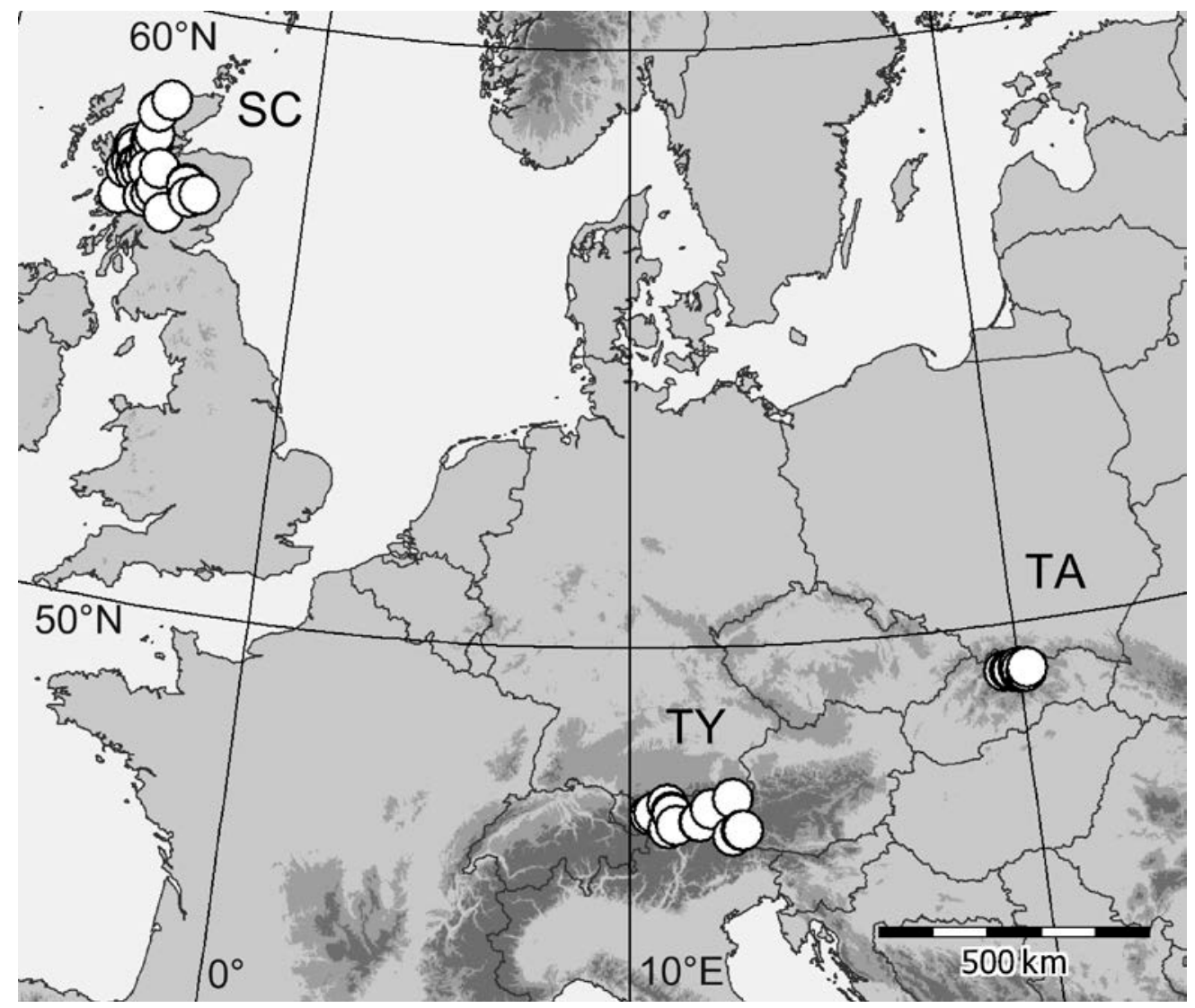

Fig 1: Map of the surveyed lakes belonging to three areas (lake districts): Scotland (SC, 30 lakes), the Alps in Tyrol in Austria (TY, 22 lakes), the Tatra Mts. in Slovakia and Poland (TA, 45 lakes). 
Table 1: List of the surveyed lakes in the Tatra Mts. (EMERGE code beginning TA) and the Alps (code TY), in Scotland (code SC) and their characteristics. DTOC - dissolved or total organic carbon concentration (DOC in the Tatra Mts. and the Alps, TOC in Scotland), Chl-a - chlorophyll-a, AA - Arctodiaptomus alpinus, AAF - Alona affinis, AE - Acroperus elongatus, AEX - Alonella excisa, AH - Acroperus harpae, AL Arctodiaptomus laticeps, AN - Alonella nana, AR - Alona rectangula, ARU - Alona rustica, AV - Acanthocyclops vernalis, BC - Bryocamptus cuspidatus, BLR - Bosmina longirostris, BLS - Bosmina longispina, BP - Branchinecta paludosa, CA - Cyclops abyssorum, CC - Chaoborus crystallinus, CO - Chaoborus obscuripes, CQ - Ceriodaphnia quadrangula, CS - Chydorus sphaericus, DB - Diaphanosoma brachyurum, DD Drepanothrix dentata, DG - Daphnia galeata, DL - Daphnia longispina, DN - Diacyclops nanus, DP - Daphnia pulicaria, DR - Daphnia rosea, EG - Eudiaptomus gracilis, EL - Eurycercus lamellatus, ES Eucyclops serrulatus, GT - Graptoleberis testudinaria, HG - Holopedium gibberum, ML - Mixodiaptomus laciniatus, MV - Megacyclops viridis, PP - Polyphemus pediculus, PPI - Paralona pigra, RF -

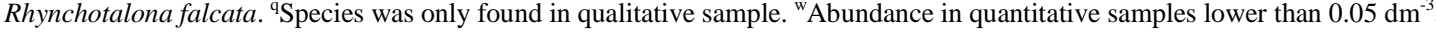

\begin{tabular}{|c|c|c|c|c|c|c|c|c|c|c|}
\hline \begin{tabular}{|c|} 
EMERGE \\
code
\end{tabular} & Name & Geographical coordinates & $\begin{array}{c}\text { Lake area } \\
\text { ha }\end{array}$ & $\begin{array}{c}\text { Depth } \\
\mathrm{m}\end{array}$ & $\begin{array}{c}\text { Altitude } \\
\mathrm{m}\end{array}$ & $\mathbf{p H}$ & $\begin{array}{c}\text { DTOC } \\
\mathrm{mg} \mathrm{C} \mathrm{dm}^{-3}\end{array}$ & $\begin{array}{c}\text { Chl-a } \\
\mu \mathrm{g} \mathrm{dm}^{-3} \\
\end{array}$ & Crustacean taxa found in zooplankton & Fish \\
\hline SC0002 & Loch Coir' a' Ghrunnda & $57^{\circ} 12^{\prime} 7^{\prime \prime} \mathrm{N}, 6^{\circ} 13^{\prime} 16^{\prime \prime} \mathrm{W}$ & 2,87 & 9,2 & 750 & 6,1 & 0,7 & 0,3 & $\mathrm{BLS} \mathrm{AE}^{\mathrm{w}}, \mathrm{RF}^{\mathrm{w}}$ & yes \\
\hline SC0010 & Lochan Bac an Lochain & $56^{\circ} 43^{\prime} 24^{\prime \prime} \mathrm{N}, 5^{\circ} 39^{\prime} 25^{\prime \prime} \mathrm{W}$ & 1,76 & 4,3 & 590 & 6,3 & 2,4 & 0,76 & DB,CQ ${ }^{q}, \mathrm{BLS}, \mathrm{AAF}^{\mathrm{q}}, \mathrm{AEX}^{\mathrm{w}}, \mathrm{AN}^{\mathrm{q}}, \mathrm{EG}, \mathrm{CA}$ & yes \\
\hline SC0029 & & $57^{\circ} 10^{\prime} 25^{\prime \prime} \mathrm{N}, 5^{\circ} 35^{\prime} 34^{\prime \prime} \mathrm{W}$ & 2,52 & 1 & 720 & 6,1 & 5,2 & 0,78 & $\mathrm{DD}, \mathrm{BLS}, \mathrm{AE}^{\mathrm{q}}, \mathrm{AH}^{\mathrm{w}}, \mathrm{AAF}^{\mathrm{q}}, \mathrm{AEX}^{\mathrm{w}}, \mathrm{AN}^{\mathrm{w}}$ & ? \\
\hline SC0067 & Loch Coire na Caime & $57^{\circ} 33^{\prime} 57 " \mathrm{~N}, 5^{\circ} 28^{\prime} 33^{\prime \prime} \mathrm{W}$ & 3,21 & 5,1 & 530 & 6 & 1 & 0,65 & $\mathrm{DD}^{\mathrm{q}}, \mathrm{DR}, \mathrm{BLS}, \mathrm{AH}^{\mathrm{w}}, \mathrm{AN}, \mathrm{GT}^{\mathrm{q}}, \mathrm{CA}$ & yes \\
\hline SC0068 & Loch Bhuic Moir & $57^{\circ} 16^{\prime} 43^{\prime \prime N}, 5^{\circ} 26^{\prime} 53^{\prime \prime} \mathrm{W}$ & 3,55 & 7,8 & 540 & 6,5 & 5,6 & 0,62 & $\mathrm{DB}^{\mathrm{w}}, \mathrm{HG}, \mathrm{BLS}, \mathrm{AH}^{\mathrm{w}}, \mathrm{AAF}^{\mathrm{q}}, \mathrm{EG}, \mathrm{CA}$ & ? \\
\hline SC0076 & Loch Coire Mhic Fhearchair & $57^{\circ} 35^{\prime} 28^{\prime \prime N}, 5^{\circ} 26^{\prime} 42^{\prime \prime} \mathrm{W}$ & 10,05 & 24 & 600 & 6,2 & 1,5 & 0,27 & $\mathrm{HG}^{\mathrm{w}}, \mathrm{BLS}, \mathrm{AE}^{\mathrm{w}}, \mathrm{AH}^{\mathrm{w}}, \mathrm{CA}$ & yes \\
\hline SC0084 & & $57^{\circ} 31^{\prime} 0^{\prime \prime} \mathrm{N}, 5^{\circ} 23^{\prime} 32^{\prime \prime} \mathrm{W}$ & 9,65 & 32 & 670 & 5,5 & 0,9 & 0,23 & $\mathrm{HG}^{\mathrm{w}}, \mathrm{BLS}, \mathrm{AE}^{\mathrm{q}}, \mathrm{AH}^{\mathrm{q}}, \mathrm{AAF}^{\mathrm{q}}, \mathrm{EG}^{\mathrm{w}}, \mathrm{CA}$ & yes \\
\hline SC0101 & Loch a' Chleirich & $57^{\circ} 15^{\prime} 3 " \mathrm{~N}, 5^{\circ} 18^{\prime} 19^{\prime \prime} \mathrm{W}$ & 1,44 & 1,7 & 750 & 6,9 & 3 & 0,88 & BLS,AH,AEX ${ }^{\mathrm{q}}, \mathrm{AN}, \mathrm{EG}, \mathrm{CA}, \mathrm{DN}, \mathrm{ES}$ & no \\
\hline SC0108 & & $57^{\circ} 8 ' 34^{\prime \prime} \mathrm{N}, 5^{\circ} 17^{\prime} 14 " \mathrm{~W}$ & 1,9 & 8,1 & 720 & 5,9 & 2 & 0,19 & BLS, $\mathrm{AH}^{\mathrm{w}}, \mathrm{PPI}^{\mathrm{q}}, \mathrm{EG}, \mathrm{CA}$ & $?$ \\
\hline SC0124 & Loch an Fhraoich-choire & $57^{\circ} 16^{\prime} 29^{\prime \prime} \mathrm{N}, 5^{\circ} 13 ' 34^{\prime \prime} \mathrm{W}$ & 4,42 & 6,2 & 660 & 6,6 & 2,4 & 0,6 & 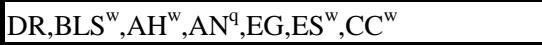 & ? \\
\hline SC0140 & Gorm Lochan & $57^{\circ} 10^{\prime} 19 " \mathrm{~N}, 5^{\circ} 6^{\prime} 13^{\prime \prime} \mathrm{W}$ & 1,64 & 9,8 & 870 & 6,8 & 1,4 & 1,96 & $\mathrm{DR}^{\mathrm{q}}, \mathrm{BLS}^{\mathrm{q}}, \mathrm{AH}^{\mathrm{q}}, \mathrm{EG}^{\mathrm{q}}$ & yes \\
\hline SC0153 & Loch Beag & $57^{\circ} 22^{\prime} 19^{\prime \prime} \mathrm{N}, 5^{\circ} 5^{\prime} 4^{\prime \prime} \mathrm{W}$ & 2,45 & 13 & 660 & 6,7 & 2,7 & 0,32 & $\mathrm{DR}, \mathrm{BLS}, \mathrm{AE}^{\mathrm{w}}, \mathrm{AH}, \mathrm{AAF}^{\mathrm{q}}, \mathrm{AN}^{\mathrm{q}}, \mathrm{EG}$ & yes \\
\hline SC0165 & Loch a' Mhadaidh & $57^{\circ} 42^{\prime} 45^{\prime \prime} \mathrm{N}, 5^{\circ} 1^{\prime} 28 " \mathrm{~W}$ & 31,1 & 46 & 570 & 5,9 & 1,9 & 0,3 & 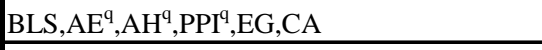 & yes \\
\hline SC0172 & & $56^{\circ} 44^{\prime} 48^{\prime \prime} \mathrm{N}, 4^{\circ} 54^{\prime} 15^{\prime \prime} \mathrm{W}$ & 9,32 & 9,1 & 740 & 6,6 & 2,6 & 1,18 & DP,BLS, $\mathrm{AE}^{\mathrm{q}}, \mathrm{AH}^{\mathrm{q}}, \mathrm{AAF}^{\mathrm{q}}, \mathrm{AEX}^{\mathrm{q}}, \mathrm{PPI}^{\mathrm{q}}, \mathrm{EG}, \mathrm{CC}$ & yes \\
\hline SC0180 & Loch Toll Lochan & $57^{\circ} 29^{\prime} 44^{\prime \prime} \mathrm{N}, 4^{\circ} 57^{\prime} 18^{\prime \prime} \mathrm{W}$ & 7,54 & 14 & 520 & 6,2 & 6,7 & 0,4 & $\mathrm{DR}, \mathrm{BLS}, \mathrm{AE}^{\mathrm{w}}, \mathrm{AH}^{\mathrm{q}}, \mathrm{AN}^{\mathrm{q}}, \mathrm{EG}, \mathrm{CA}$ & yes \\
\hline SC0189 & Loch an Fhuar-thuill Mhoir & $57^{\circ} 27^{\prime} 9 " \mathrm{~N}, 4^{\circ} 56^{\prime} 12^{\prime \prime} \mathrm{W}$ & 5,36 & 15 & 770 & 6,2 & 0,7 & 0,36 & DR,BLS, $\mathrm{AE}^{\mathrm{q}}, \mathrm{AN}^{\mathrm{q}}, \mathrm{AL}, \mathrm{CA}$ & ? \\
\hline SC0190 & Loch Gorm & $57^{\circ} 40^{\prime} 53^{\prime \prime} \mathrm{N}, 4^{\circ} 57^{\prime} 16^{\prime \prime} \mathrm{W}$ & 21,61 & 47 & 540 & 6,2 & 2 & 0,44 & $\mathrm{DR}, \mathrm{BLS}, \mathrm{AH}^{\mathrm{w}}, \mathrm{AL}, \mathrm{CA}$ & yes \\
\hline SC0191 & Loch Carn a' Chaochain & $57^{\circ} 13^{\prime} 13^{\prime \prime} \mathrm{N}, 4^{\circ} 54^{\prime} 56^{\prime \prime} \mathrm{W}$ & 2,29 & 5 & 660 & 6,2 & 6,7 & 2,04 & $\mathrm{DB}^{\mathrm{w}}, \mathrm{BLS}, \mathrm{AEX}^{\mathrm{w}}, \mathrm{AN}^{\mathrm{w}}, \mathrm{ML}$ & $?$ \\
\hline SC0197 & Loch a' Choire Dhairg & $58^{\circ} 11^{\prime} 57 " \mathrm{~N}, 4^{\circ} 58^{\prime} 35^{\prime \prime} \mathrm{W}$ & 4,22 & 11,5 & 530 & 6,8 & 1,7 & 0,63 & $\mathrm{HG}, \mathrm{DR}, \mathrm{BLS}, \mathrm{AE}^{\mathrm{q}}, \mathrm{AH}^{\mathrm{w}}, \mathrm{AAF}^{\mathrm{q}}, \mathrm{PPI}^{\mathrm{q}}, \mathrm{CA}$ & yes \\
\hline SC0204 & Lochan a' Chnapaich & $57^{\circ} 48^{\prime} 35^{\prime \prime} \mathrm{N}, 4^{\circ} 56^{\prime} 8^{\prime \prime} \mathrm{W}$ & 5,6 & 8,8 & 690 & 6,5 & 2,4 & 0,36 & $\mathrm{DR}, \mathrm{BLS}, \mathrm{AH}^{\mathrm{w}}{ }^{\mathrm{w}} \mathrm{EG}, \mathrm{ML}$ & ? \\
\hline SC0211 & Loch Bealach na h-Uidhe & $58^{\circ} 11^{\prime} 6 " \mathrm{~N}, 4^{\circ} 57^{\prime} 13^{\prime \prime} \mathrm{W}$ & 2,9 & 3,5 & 530 & 6,2 & 3,5 & 0,83 & DR,BLS,AH,ML,CA & yes \\
\hline SC0271 & Lochan Coire an Lochain & $56^{\circ} 49^{\prime} 53^{\prime \prime} \mathrm{N}, 4^{\circ} 41^{\prime} 3^{\prime \prime} \mathrm{W}$ & 5,06 & 4,7 & 740 & 6,9 & 3,2 & 0,37 & $\mathrm{HG}, \mathrm{BLS}, \mathrm{AE}^{\mathrm{w}}, \mathrm{AH}^{\mathrm{w}}, \mathrm{AN}^{\mathrm{q}}, \mathrm{MV}^{\mathrm{w}}$ & yes \\
\hline SC0330 & Lochan Coire Choille-rais & $56^{\circ} 56^{\prime} 43^{\prime \prime} \mathrm{N}, 4^{\circ} 34^{\prime} 37^{\prime \prime} \mathrm{W}$ & 7,4 & 27 & 810 & 6,7 & 1,3 & 0,41 & $\mathrm{HG}^{\mathrm{q}}, \mathrm{DR}, \mathrm{BLS}, \mathrm{AH}^{\mathrm{w}}, \mathrm{AAF}^{\mathrm{q}}, \mathrm{CA}$ & ? \\
\hline SC0335 & & $57^{\circ} 16^{\prime} 9 " \mathrm{~N}, 4^{\circ} 34^{\prime} 58^{\prime \prime} \mathrm{W}$ & 1,92 & 6 & 540 & 6,8 & 9,2 & 0,44 & $\mathrm{DB}^{\mathrm{q}}, \mathrm{DL}^{\mathrm{q}}, \mathrm{BLS}, \mathrm{AH}^{\mathrm{q}}, \mathrm{AAF}^{\mathrm{q}}, \mathrm{AEX}^{\mathrm{w}}, \mathrm{AL}, \mathrm{CA}, \mathrm{ES}^{\mathrm{w}}$ & ? \\
\hline SC0349 & & $58^{\circ} 25^{\prime} 1 " \mathrm{~N}, 4^{\circ} 35^{\prime} 43^{\prime \prime} \mathrm{W}$ & 3,82 & 7,3 & 530 & 6 & 2,6 & 0,63 & DR, $\mathrm{BLS}^{\mathrm{q}}, \mathrm{AH}^{\mathrm{q}}, \mathrm{AN}^{\mathrm{q}}, \mathrm{ML}^{\mathrm{w}}, \mathrm{CA}^{\mathrm{q}}, \mathrm{CC}^{\mathrm{w}}$ & ? \\
\hline SC0366 & Lochan nan Cat & $56^{\circ} 33^{\prime} 22^{\prime \prime} \mathrm{N}, 4^{\circ} 12^{\prime} 23^{\prime \prime} \mathrm{W}$ & 12,37 & 14 & 720 & 7,1 & 2,9 & & $\mathrm{HG}, \mathrm{DG}, \mathrm{BLS}, \mathrm{AE}^{\mathrm{q}}, \mathrm{AH}^{\mathrm{w}}, \mathrm{AN}^{\mathrm{q}}, \mathrm{MV}$ & yes \\
\hline SC0379 & & $57^{\circ} 6^{\prime} 20^{\prime \prime} \mathrm{N}, 3^{\circ} 40^{\prime} 60^{\prime \prime} \mathrm{W}$ & 0,91 & 5,6 & 920 & 5,8 & 0,8 & 0,14 & $\mathrm{DR}^{\mathrm{w}}, \mathrm{BLS} \mathrm{AH}^{\mathrm{w}}, \mathrm{ARU}^{\mathrm{w}}, \mathrm{CS}^{\mathrm{w}}, \mathrm{EG}, \mathrm{ML}, \mathrm{CA}, \mathrm{ES}^{\mathrm{w}}$ & no \\
\hline SC0382 & Lochan Uaine & $57^{\circ} 3^{\prime} 47^{\prime \prime} \mathrm{N}, 3^{\circ} 38^{\prime} 55^{\prime \prime} \mathrm{W}$ & 3,78 & 21 & 910 & 5,9 & 1,1 & 0,31 & HG,BLS, $\mathrm{AE}^{\mathrm{w}}, \mathrm{AH}^{\mathrm{w}}, \mathrm{CS}^{\mathrm{q}}, \mathrm{MV}^{\mathrm{w}}$ & no \\
\hline
\end{tabular}




\begin{tabular}{|c|c|c|c|c|c|c|c|c|c|c|}
\hline \begin{tabular}{|c|} 
EMERGE \\
code
\end{tabular} & Name & Geographical coordinates & $\begin{array}{c}\text { Lake area } \\
\text { ha }\end{array}$ & $\begin{array}{c}\text { Depth } \\
\mathrm{m}\end{array}$ & $\begin{array}{c}\text { Altitude } \\
\mathrm{m}\end{array}$ & $\mathbf{p H}$ & \begin{tabular}{|c|} 
DTOC \\
$\mathrm{mg} \mathrm{C} \mathrm{dm}^{-3}$ \\
\end{tabular} & \begin{tabular}{|c|} 
Chl-a \\
$\mu \mathrm{g} \mathrm{dm}^{-3}$ \\
\end{tabular} & Crustacean taxa found in zooplankton & Fish \\
\hline SC0386 & Loch nan Eun & $56^{\circ} 53^{\prime} 6^{\prime \prime} \mathrm{N}, 3^{\circ} 32^{\prime} 18^{\prime \prime} \mathrm{W}$ & 14,46 & 10 & 790 & 6,7 & 5,1 & 1,6 & DR,BLS,EL ${ }^{\mathrm{q}}, \mathrm{PPI}^{\mathrm{q}}, \mathrm{EG}, \mathrm{ML}, \mathrm{CA}, \mathrm{ES}^{\mathrm{q}}$ & yes \\
\hline SC0399 & Lochnagar & $56^{\circ} 57^{\prime} 33^{\prime \prime} \mathrm{N}, 3^{\circ} 13^{\prime} 53^{\prime \prime} \mathrm{W}$ & 9,9 & 24 & 790 & 5,4 & 1,6 & 0,47 & $\mathrm{HG}, \mathrm{BLS}, \mathrm{AH}^{\mathrm{w}}{ }^{\mathrm{w}} \mathrm{EG}$ & yes \\
\hline TA0001 & Horné Roháčske pleso & $49^{\circ} 12^{\prime} 21^{\prime \prime} \mathrm{N}, 19^{\circ} 37^{\prime} 37^{\prime \prime} \mathrm{E}$ & 1,45 & 8,1 & 1718 & 6,4 & 0,44 & 0,72 & $\mathrm{DR}, \mathrm{AH}, \mathrm{AAF}^{\mathrm{q}}, \mathrm{AV}^{\mathrm{q}}$ & no \\
\hline TA0003 & Nižné Jamnícke pleso & $49^{\circ} 12^{\prime} 11^{\prime \prime} \mathrm{N}, 19^{\circ} 46^{\prime} 18^{\prime \prime} \mathrm{E}$ & 1,12 & 9,2 & 1728 & 7,2 & 0,43 & 0,67 & $\mathrm{DR}, \mathrm{AH}^{\mathrm{w}}, \mathrm{AAF}^{\mathrm{q}}, \mathrm{CA}, \mathrm{ES}^{\mathrm{q}}$ & no \\
\hline TA0004 & Vyšné Račkove pleso & $49^{\circ} 12^{\prime} 0 " \mathrm{~N}, 19^{\circ} 48^{\prime} 23^{\prime \prime} \mathrm{E}$ & 0,73 & 12,7 & 1697 & 7,2 & 0,13 & 0,65 & DR,AAF ${ }^{\mathrm{q}}, \mathrm{CS}, \mathrm{CA}$ & yes \\
\hline TA0006 & Vel'ké Bystré pleso & $49^{\circ} 10^{\prime} 59^{\prime \prime} \mathrm{N}, 19^{\circ} 50^{\prime} 37^{\prime \prime} \mathrm{E}$ & 0,89 & 12,7 & 1876 & 7,3 & 1 & 0,55 & CA & yes \\
\hline TA0007 & Zielony Staw Gąsienicowy & $49^{\circ} 13^{\prime} 44^{\prime \prime} \mathrm{N}, 20^{\circ} 0^{\prime} 4^{\prime \prime} \mathrm{E}$ & 3,84 & 15,1 & 1672 & 6,8 & 0,67 & 5,75 & $\mathrm{AH}^{\mathrm{w}}, \mathrm{AAF}^{\mathrm{w}}, \mathrm{CA}$ & yes \\
\hline TA0008 & Zelené krivánske pleso & $49^{\circ} 9^{\prime} 34 " \mathrm{~N}, 20^{\circ} 0^{\prime} 31^{\prime \prime} \mathrm{E}$ & 4,32 & 23,1 & 2017 & 6,7 & 0,274 & 1,4 & DP,CS,CA & no \\
\hline TA0009 & Długi Staw Gąsienicowy & $49^{\circ} 13^{\prime} 38^{\prime \prime} \mathrm{N}, 20^{\circ} 0^{\prime} 39^{\prime \prime} \mathrm{E}$ & 1,58 & 10,6 & 1784 & 6,2 & 0,223 & 0,11 & $\mathrm{CS}, \mathrm{AV}^{\mathrm{W}}$ & no \\
\hline TA0010 & Zadni Staw Gąsienicowy & $49^{\circ} 13^{\prime} 32^{\prime \prime} \mathrm{N}, 20^{\circ} 0^{\prime} 43^{\prime \prime} \mathrm{E}$ & 0,53 & 8 & 1852 & 6,4 & 0,234 & 0,03 & $\mathrm{CS}, \mathrm{AV}^{\mathrm{w}}$ & no \\
\hline TA0011 & Nižné Terianske pleso & $49^{\circ} 10^{\prime} 11^{\prime \prime} \mathrm{N}, 20^{\circ} 0^{\prime} 51 " \mathrm{E}$ & 4,91 & 43,2 & 1941 & 6,7 & 0,255 & 2,4 & $\mathrm{CS}^{\mathrm{w}}, \mathrm{CA}, \mathrm{ES}^{\mathrm{q}}$ & no \\
\hline TA0012 & Zadni Staw Polski & $49^{\circ} 12^{\prime} 48^{\prime \prime} \mathrm{N}, 20^{\circ} 0^{\prime} 511^{\prime \prime} \mathrm{E}$ & 6,46 & 31,6 & 1890 & 6,3 & 0,446 & 0,57 & $\mathrm{AAF}^{\mathrm{w}}, \mathrm{CS}^{\mathrm{w}}, \mathrm{CA}, \mathrm{ES}^{\mathrm{q}}$ & no \\
\hline TA0013 & Czarny Staw Gąsienicowy & $49^{\circ} 13^{\prime} 52^{\prime \prime} \mathrm{N}, 20^{\circ} 1^{\prime} 12^{\prime \prime} \mathrm{E}$ & 17,79 & 51 & 1620 & 6,4 & 0,51 & 3,09 & $\mathrm{AH}^{\mathrm{q}}, \mathrm{AAF}^{\mathrm{w}}, \mathrm{CA}$ & yes \\
\hline TA0014 & Vyšné Terianske pleso & $49^{\circ} 10^{\prime} 5 " \mathrm{~N}, 20^{\circ} 1{ }^{\prime} 18^{\prime \prime} \mathrm{E}$ & 0,55 & 4,2 & 2109 & 5 & 0,554 & 1,83 & $\mathrm{CS}, \mathrm{AV}$ & no \\
\hline TA0015 & Zmarzly Staw Gąsienicowy & $49^{\circ} 13^{\prime} 28^{\prime \prime} \mathrm{N}, 20^{\circ} 1^{\prime} 26^{\prime \prime} \mathrm{E}$ & 0,28 & 3,7 & 1787 & 6 & 0,14 & 0,05 & $\mathrm{CS}^{\mathrm{w}}, \mathrm{AV}^{\mathrm{q}}$ & no \\
\hline TA0017 & Vyšné Wahlenbergovo pleso & $49^{\circ} 9^{\prime} 51 " \mathrm{~N}, 20^{\circ} 18^{\prime \prime} \mathrm{E}$ & 4,96 & 21,1 & 2145 & 6,3 & 0,15 & 1,23 & $\mathrm{CS}^{\mathrm{w}}, \mathrm{CA}, \mathrm{ES}^{\mathrm{w}}$ & no \\
\hline TA0018 & Czarny Staw Polski & $49^{\circ} 12^{\prime} 17^{\prime \prime} \mathrm{N}, 20^{\circ} 1^{\prime} 40^{\prime \prime} \mathrm{E}$ & 12,65 & 50,4 & 1722 & 6,6 & 0,95 & 3,84 & $\mathrm{CS}^{\mathrm{W}}, \mathrm{CA}$ & yes \\
\hline TA0019 & Nižné Temnosmrečinské pleso & $49^{\circ} 11^{\prime} 34^{\prime \prime} \mathrm{N}, 20^{\circ} 1^{\prime} 50^{\prime \prime} \mathrm{E}$ & 10,48 & 40,5 & 1674 & 7,2 & 0,354 & 0,83 & DG,DP,CS ${ }^{\mathrm{w}}, \mathrm{CA}$ & no \\
\hline TA0020 & Okrúhle pleso & $49^{\circ} 10^{\prime} 15^{\prime \prime} \mathrm{N}, 20^{\circ} 2^{\prime} 11^{\prime \prime} \mathrm{E}$ & 0,75 & 10,2 & 2096 & 6,1 & 0,27 & 0,39 & AA & no \\
\hline TA0021 & Capie pleso & $49^{\circ} 10^{\prime} 6 " \mathrm{~N}, 20^{\circ} 2^{\prime} 16^{\prime \prime} \mathrm{E}$ & 2,43 & 16,8 & 2072 & 6,3 & 0,22 & 0,96 & $\mathrm{CS}^{\mathrm{w}}, \mathrm{AA}^{\mathrm{w}}, \mathrm{CA}^{\mathrm{w}}, \mathrm{ES}$ & no \\
\hline TA0022 & Vyšné Temnosmrečinské pleso & $49^{\circ} 11^{\prime} 21 " \mathrm{~N}, 20^{\circ} 2^{\prime 2} 22^{\prime \prime} \mathrm{E}$ & 4,95 & 20 & 1716 & 7,2 & 0,204 & 1,43 & $\mathrm{DP}, \mathrm{CS}^{\mathrm{w}}, \mathrm{CA}$ & no \\
\hline TA0023 & Wielki Staw Polski & $49^{\circ} 12^{\prime} 48^{\prime \prime} \mathrm{N}, 20^{\circ} 2^{\prime} 25^{\prime \prime} \mathrm{E}$ & 34,14 & 79,3 & 1655 & 6,7 & 0,41 & 0,61 & DP,CA & yes \\
\hline TA0026 & Malé Hincovo pleso & $49^{\circ} 10^{\prime} 26^{\prime \prime} \mathrm{N}, 20^{\circ} 3^{\prime} 31^{\prime \prime} \mathrm{E}$ & 2,22 & 6,1 & 1923 & 7,3 & 0,58 & 0,86 & $\mathrm{DP}^{\mathrm{w}}, \mathrm{AH}^{\mathrm{w}}, \mathrm{CS}^{\mathrm{w}}, \mathrm{AA}, \mathrm{CA}, \mathrm{ES}^{\mathrm{w}}$ & no \\
\hline TA0027 & Vel'ké Hincovo pleso & $49^{\circ} 10^{\prime} 47^{\prime \prime} \mathrm{N}, 20^{\circ} 3^{\prime} 38^{\prime \prime} \mathrm{E}$ & 18,19 & 53,2 & 1946 & 6,9 & 0,42 & 1,14 & $\mathrm{AH}^{\mathrm{w}}, \mathrm{AAF}^{\mathrm{w}}, \mathrm{CS}^{\mathrm{w}}, \mathrm{AA}, \mathrm{CA}$ & yes \\
\hline TA0029 & Czarny Staw pod Rysami & $49^{\circ} 11^{\prime} 20^{\prime \prime N}, 20^{\circ} 4^{\prime} 40 " \mathrm{E}$ & 20,54 & 76,4 & 1580 & 7 & 0,264 & 0,96 & DP,CA & no \\
\hline TA0030 & Vel'ké Žabie pleso & $49^{\circ} 10^{\prime} 19^{\prime \prime} \mathrm{N}, 20^{\circ} 4^{\prime} 43^{\prime \prime} \mathrm{E}$ & 2,26 & 6,9 & 1919 & 6,5 & 0,15 & 0,75 & $\mathrm{CS}, \mathrm{ES}^{\mathrm{w}}$ & no \\
\hline TA0031 & Dračie pleso & $49^{\circ} 9^{\prime} 59^{\prime \prime} \mathrm{N}, 20^{\circ} 5^{\prime} 15^{\prime \prime} \mathrm{E}$ & 1,71 & 16 & 1998 & 6,9 & 0,05 & 0,41 & $\mathrm{AA}$ & no \\
\hline TA0032 & Vyšné Žabie bielovodské pleso & $49^{\circ} 11^{\prime} 39^{\prime \prime} \mathrm{N}, 20^{\circ} 5^{\prime} 39^{\prime \prime} \mathrm{E}$ & 8,08 & 24,3 & 1699 & 6,6 & 0,43 & 0,53 & DP, $\mathrm{AAF}^{\mathrm{w}}, \mathrm{CS}^{\mathrm{w}}, \mathrm{CA}$ & no \\
\hline TA0036 & Litvorové pleso & $49^{\circ} 10^{\prime} 38^{\prime \prime} \mathrm{N}, 20^{\circ} 7^{\prime} 52^{\prime \prime} \mathrm{E}$ & 1,67 & 18,6 & 1863 & 6,9 & 0,14 & 1,18 & $\mathrm{DP}, \mathrm{AH}^{\mathrm{q}}, \mathrm{CA}$ & no \\
\hline TA0037 & Batizovské pleso & $49^{\circ} 9^{\prime} 8 " \mathrm{~N}, 20^{\circ} 7^{\prime} 53^{\prime \prime} \mathrm{E}$ & 2,78 & 11,2 & 1879 & 6,3 & 0,1 & 0,57 & $\mathrm{CS}^{\mathrm{w}}$ & no \\
\hline TA0039 & Zelené javorové pleso & $49^{\circ} 12^{\prime} 22^{\prime \prime} \mathrm{N}, 20^{\circ} 8^{\prime} 34^{\prime \prime} \mathrm{E}$ & 0,75 & 8,3 & 1811 & 6,6 & 0,36 & 1,28 & DP,CS,AA,CA & no \\
\hline TA0042 & Pusté pleso & $49^{\circ} 10^{\prime} 56^{\prime \prime} \mathrm{N}, 20^{\circ} 9^{\prime} 18^{\prime \prime} \mathrm{E}$ & 1,2 & 6,82 & 2055 & 6,9 & 0,28 & 0,84 & $\mathrm{CS}^{\mathrm{w}}, \mathrm{AA}, \mathrm{CA}^{\mathrm{q}}$ & no \\
\hline TA0043 & Vyšné zbojnícke pleso & $49^{\circ} 10^{\prime} 44^{\prime \prime} \mathrm{N}, 20^{\circ} 9^{\prime} 34^{\prime \prime} \mathrm{E}$ & 0,66 & 7 & 1972 & 6,9 & 0,27 & 0,39 & $\mathrm{CS}^{\mathrm{w}}, \mathrm{AA}$ & no \\
\hline TA0044 & Prostredné zbojnícke pleso & $49^{\circ} 10^{\prime} 42^{\prime \prime} \mathrm{N}, 20^{\circ} 9^{\prime} 42^{\prime \prime} \mathrm{E}$ & 0,62 & 5,4 & 1969 & 6,8 & 0,31 & 0,32 & $\mathrm{AA}, \mathrm{AV}^{\mathrm{q}}, \mathrm{CA}^{\mathrm{w}}$ & no \\
\hline
\end{tabular}




\begin{tabular}{|c|c|c|c|c|c|c|c|c|c|c|}
\hline \begin{tabular}{|c|} 
EMERGE \\
code
\end{tabular} & Name & Geographical coordinates & $\begin{array}{c}\text { Lake area } \\
\text { ha }\end{array}$ & $\begin{array}{c}\text { Depth } \\
\mathrm{m}\end{array}$ & $\begin{array}{c}\text { Altitude } \\
\text { m }\end{array}$ & $\mathbf{p H}$ & $\begin{array}{c}\text { DTOC } \\
\mathrm{mg} \mathrm{C} \mathrm{dm}^{-3}\end{array}$ & $\begin{array}{c}\text { Chl-a } \\
\mu \mathrm{g} \mathrm{dm}^{-3}\end{array}$ & Crustacean taxa found in zooplankton & Fish \\
\hline TA0045 & Ladové pleso & $49^{\circ} 11^{\prime} 3 " \mathrm{~N}, 20^{\circ} 9^{\prime} 46^{\prime \prime} \mathrm{E}$ & 1,72 & 18 & 2057 & 6,6 & 0,33 & 0,69 & CS,AA,CA & no \\
\hline TA0047 & Starolesnianske pleso & $49^{\circ} 10^{\prime} 48^{\prime \prime N}, 20^{\circ} 10^{\prime} 4 " \mathrm{E}$ & 0,73 & 4,1 & 1986 & 5,3 & 1,37 & 5,26 & $\mathrm{CQ}^{\mathrm{w}}, \mathrm{CS}, \mathrm{AV}$ & no \\
\hline TA0049 & Žabie javorové pleso & $49^{\circ} 11^{\prime} 28^{\prime \prime} \mathrm{N}, 20^{\circ} 10^{\prime} 12^{\prime \prime} \mathrm{E}$ & 0,83 & 16 & 1886 & 7 & 0,1 & 0,33 & DP,CA & no \\
\hline TA0051 & Prostredné sivé pleso & $49^{\circ} 11^{\prime} 3 " \mathrm{~N}, 20^{\circ} 10^{\prime} 36^{\prime \prime} \mathrm{E}$ & 0,88 & 5,2 & 2011 & 5,8 & 0,33 & 0,04 & $\mathrm{CS}^{\mathrm{w}}, \mathrm{BC}$ & no \\
\hline TA0054 & Vel'ké spišské pleso & $49^{\circ} 11^{\prime} 36^{\prime \prime N}, 20^{\circ} 11^{\prime} 47^{\prime \prime E}$ & 2,43 & 9,6 & 2014 & 6,2 & 0,056 & 0,14 & & no \\
\hline TA0056 & Prostredné spišské pleso & $49^{\circ} 11^{\prime} 29 " \mathrm{~N}, 20^{\circ} 11^{\prime} 56^{\prime \prime} \mathrm{E}$ & 1,82 & 5,1 & 2013 & 6 & 0,141 & 1,42 & $\mathrm{CS}$ & no \\
\hline TA0101 & Wyżni Siwy Stawek & $49^{\circ} 12^{\prime} 18^{\prime \prime N}, 19^{\circ} 50^{\prime} 14^{\prime \prime} \mathrm{E}$ & 0,05 & 1,8 & 1716 & 7,2 & 3,33 & 0,27 & $\mathrm{CS}^{\mathrm{w}}$ & no \\
\hline TA0102 & Dwoisty Staw Wschodni & $49^{\circ} 13^{\prime} 60^{\prime \prime} \mathrm{N}, 20^{\circ} 0^{\prime} 25^{\prime \prime} \mathrm{E}$ & 1,41 & 9,2 & 1657 & 5,8 & 0,23 & 0,95 & $\mathrm{HG}, \mathrm{AH}, \mathrm{EL}^{\mathrm{q}}, \mathrm{CS}^{\mathrm{w}}, \mathrm{ES}^{\mathrm{q}}$ & no \\
\hline TA0105 & Vyšné Furkotské pleso & $49^{\circ} 8 ' 37^{\prime \prime N}, 20^{\circ} 1 ' 53 " \mathrm{E}$ & 0,46 & 3,4 & 1698 & 6,5 & 0,32 & 1,04 & $\mathrm{BP}, \mathrm{DR}, \mathrm{AR}^{\mathrm{q}}, \mathrm{CS}, \mathrm{AA}$ & no \\
\hline TA0106 & Wyżni Mnichowy Stawek IX & $49^{\circ} 11^{\prime} 42^{\prime \prime} \mathrm{N}, 20^{\circ} 3^{\prime} 17^{\prime \prime} \mathrm{E}$ & 0,06 & 2,3 & 1870 & 4,8 & 0,74 & 1,75 & AAF,CS & no \\
\hline TA0108 & Morskie Oko & $49^{\circ} 11^{\prime} 52^{\prime \prime} \mathrm{N}, 20^{\circ} 4^{\prime} 20^{\prime \prime} \mathrm{E}$ & 34,54 & 50,8 & 1395 & 6,9 & 0,47 & 1,38 & HG,DG,BLR,PP,CA ${ }^{\mathrm{q}}, \mathrm{ES}^{\mathrm{w}}$ & yes \\
\hline TA0109 & Slavkovské pleso & $49^{\circ} 9^{\prime} 9^{\prime \prime} \mathrm{N}, 20^{\circ} 11^{\prime} 5 " \mathrm{E}$ & 0,1 & 2,91 & 1676 & 5 & 5,15 & 11,17 & 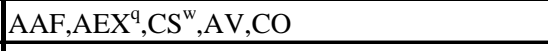 & no \\
\hline TA0110 & Čierne pleso kežmarské & $49^{\circ} 12^{\prime} 28^{\prime \prime} \mathrm{N}, 20^{\circ} 13^{\prime} 35^{\prime \prime} \mathrm{E}$ & 0,31 & 3,6 & 1579 & 7 & 0,35 & 0,97 & AAF, $\mathrm{AEX}^{\mathrm{q}}, \mathrm{CS}^{\mathrm{w}}, \mathrm{AA}$ & no \\
\hline TY0047 & Unterer Seewisee & $47^{\circ} 11^{\prime} 38^{\prime \prime N}, 10^{\circ} 28^{\prime} 55^{\prime \prime E}$ & 1,78 & 2,2 & 2229 & 8,8 & 1,644 & 0,49 & $\mathrm{AAF}^{\mathrm{q}}, \mathrm{CS}^{\mathrm{q}}, \mathrm{AA}, \mathrm{CA}^{\mathrm{q}}, \mathrm{ES}^{\mathrm{q}}$ & no \\
\hline TY0048 & Oberer Seewisee & $47^{\circ} 11^{\prime} 16 " \mathrm{~N}, 10^{\circ} 29^{\prime} 9 " \mathrm{E}$ & 1,43 & 13,5 & 2469 & 8,1 & 0,286 & 0,45 & $\mathrm{CS}^{\mathrm{q}}, \mathrm{AA}, \mathrm{CA}^{\mathrm{w}}$ & no \\
\hline TY0049 & Mittlerer Seewisee & $47^{\circ} 11^{\prime} 27^{\prime \prime N}, 10^{\circ} 29^{\prime} 26^{\prime \prime} \mathrm{E}$ & 0,52 & 4,7 & 2425 & 8,3 & 0,355 & 0,24 & & no \\
\hline TY0077 & Steinsee & $47^{\circ} 13 ' 31 " \mathrm{~N}, 10^{\circ} 35^{\prime} 47^{\prime \prime} \mathrm{E}$ & 1,87 & 8,3 & 2222 & 8,6 & 0,708 & 0,73 & $\mathrm{DR}, \mathrm{AH}^{\mathrm{w}}, \mathrm{CS}, \mathrm{AA}, \mathrm{CA}^{\mathrm{w}}$ & yes \\
\hline TY0166 & Drachensee & $47^{\circ} 21^{\prime} 15^{\prime \prime} \mathrm{N}, 10^{\circ} 56^{\prime} 5 " \mathrm{E}$ & 5,01 & 24 & 1874 & 8,3 & 1,054 & 2,12 & $\mathrm{AV}^{\mathrm{W}}$ & yes \\
\hline TY0170 & Schwarzsee ob Sölden & $46^{\circ} 57^{\prime} 42^{\prime \prime N}, 10^{\circ} 56^{\prime} 46^{\prime \prime} \mathrm{E}$ & 3,72 & 18 & 2796 & 5,7 & 0,542 & 1,61 & $\mathrm{CS}, \mathrm{AV}$ & yes \\
\hline TY0188 & Gossenköllesee & $47^{\circ} 13^{\prime} 31^{\prime \prime} \mathrm{N}, 11^{\circ} 0^{\prime} 50^{\prime \prime} \mathrm{E}$ & 1,59 & 9,9 & 2413 & 7 & 0,398 & 2,89 & $\mathrm{AEX}^{\mathrm{w}}, \mathrm{CS}, \mathrm{CA}$ & yes \\
\hline TY0189 & Rotfelssee & $47^{\circ} 13^{\prime} 35^{\prime \prime} \mathrm{N}, 11^{\circ} 0^{\prime} 29^{\prime \prime} \mathrm{E}$ & 1,01 & 5 & 2485 & 7,3 & 0,354 & 0,53 & $\mathrm{CS}^{\mathrm{w}}, \mathrm{AV}^{\mathrm{q}}$ & yes \\
\hline TY0194 & Oberer Plenderlessee & $47^{\circ} 11^{\prime} 56^{\prime \prime N}, 11^{\circ} 2^{\prime} 17^{\prime \prime E}$ & 2,23 & 7,5 & 2344 & 7,1 & 0,248 & 0,88 & CA & yes \\
\hline TY0195 & Mittlerer Plenderlessee & $47^{\circ} 12^{\prime} 17^{\prime \prime N}, 11^{\circ} 2^{\prime} 30^{\prime \prime E}$ & 1,46 & 5,76 & 2317 & 6,5 & 0,766 & 1,26 & $\mathrm{AEX}^{\mathrm{w}}, \mathrm{CA}$ & yes \\
\hline TY0229 & Mutterbergersee & $47^{\circ} 0^{\prime} 43^{\prime \prime} \mathrm{N}, 11^{\circ} 7^{\prime} 43^{\prime \prime} \mathrm{E}$ & 3,07 & 8,1 & 2479 & 5,7 & 0,44 & 0,42 & $\mathrm{CS}^{\mathrm{w}}, \mathrm{CA}$ & yes \\
\hline TY0303 & Friesenbergsee & $47^{\circ} 3^{\prime} 53^{\prime \prime} \mathrm{N}, 11^{\circ} 41^{\prime} 60^{\prime \prime} \mathrm{E}$ & 1,88 & 4,5 & 2450 & 7,6 & 0,32 & 0,21 & $\mathrm{ES}^{\mathrm{q}}$ & ? \\
\hline TY0305 & Oberer Wesendlekarsee & $47^{\circ} 4{ }^{\prime} 21^{\prime \prime} \mathrm{N}, 11^{\circ} 43^{\prime} 1 " \mathrm{E}$ & 1,72 & 16,5 & 2660 & 7,3 & 0,415 & 1,99 & $\mathrm{AEX}^{\mathrm{q}}$ & $?$ \\
\hline TY0306 & Unterer Wesendlekarsee & $47^{\circ} 43^{\prime \prime} \mathrm{N}, 11^{\circ} 43^{\prime} 11^{\prime \prime E}$ & 2,46 & 21,5 & 2368 & 7,2 & 0,624 & 1,33 & & yes \\
\hline TY0338 & Vorderer Langersee in der Wilden Krimml & $47^{\circ} 15^{\prime} 40^{\prime \prime} \mathrm{N}, 11^{\circ} 59^{\prime} 17^{\prime \prime} \mathrm{E}$ & 3,65 & 15,4 & 2232 & 7,1 & 1,639 & 7,49 & BLR,CS ${ }^{q}, C A$ & yes \\
\hline TY0339 & Oberer Scheibensee & $47^{\circ} 15^{\prime} 46^{\prime \prime} \mathrm{N}, 11^{\circ} 58^{\prime} 54^{\prime \prime} \mathrm{E}$ & 1,53 & 4 & 2290 & 7,5 & 0,861 & 1,49 & $\mathrm{BLR} \mathrm{AH}^{\mathrm{q}}, \mathrm{CS}^{\mathrm{q}}, \mathrm{AV}^{\mathrm{q}}$ & yes \\
\hline TY0427 & Aschersee & $46^{\circ} 48^{\prime} 10^{\prime \prime} \mathrm{N}, 12^{\circ} 30^{\prime} 11^{\prime \prime} \mathrm{E}$ & 1,38 & 7 & 2532 & 7 & 1,027 & 0,49 & DR,AEX ${ }^{\mathrm{q}}, \mathrm{CA}$ & yes \\
\hline TY0428 & Anraser See & $46^{\circ} 48^{\prime} 25^{\prime \prime} \mathrm{N}, 12^{\circ} 31^{\prime} 4^{\prime \prime} \mathrm{E}$ & 2,07 & 6,5 & 2538 & 7,2 & 0,779 & 0,95 & DR,AAF ${ }^{\mathrm{q}}, \mathrm{AA}, \mathrm{CA}$ & yes \\
\hline TY0444 & Wildsee ob Fieberbrunn & $47^{\circ} 25^{\prime} 36^{\prime \prime N}, 12^{\circ} 32^{\prime} 21 " \mathrm{E}$ & 3,23 & 14,5 & 1840 & 7,4 & 0,867 & 0,78 & DR,CA,ES ${ }^{\mathrm{q}}$ & yes \\
\hline TY0459 & Alkusersee & $46^{\circ} 54^{\prime} 1 " \mathrm{~N}, 12^{\circ} 43^{\prime} 59 " \mathrm{E}$ & 7,19 & 44,6 & 2432 & 7,1 & 0,416 & 2,68 & BLR,AV ${ }^{q}$ & yes \\
\hline TY0463 & Südlicher Neualplsee & $46^{\circ} 53^{\prime} 17 " \mathrm{~N}, 12^{\circ} 45^{\prime} 57^{\prime \prime} \mathrm{E}$ & 1,95 & 13,2 & 2438 & 7 & 0,862 & 18,65 & $\mathrm{AAF}^{\mathrm{w}}, \mathrm{AEX}^{\mathrm{w}}, \mathrm{AV}^{\mathrm{q}}$ & yes \\
\hline TY0464 & Nördlicher Neualplsee & $46^{\circ} 53^{\prime} 24^{\prime \prime N}, 12^{\circ} 46^{\prime} 3^{\prime \prime E}$ & 2,08 & 7,2 & 2440 & 7,1 & 1,031 & 1,36 & BLR,CA & yes \\
\hline
\end{tabular}


Organisms were identified using keys by Bartoš (1959), Šrámek-Hušek et al. (1962), Ruttner-Kolisko (1972), Kiefer (1978), Koste (1978), Rozkošný (1980), Negrea (1983), Einsle (1993), Segers (1995), De Smet (1997), and Flössner (2000). The identity of a misidentified Daphnia species from the Nižné Temnosmrečinské Lake was corrected to D. galeata according to the genetical study by Petrusek et al. (2007). The harpacticoid copepod Bryocamptus cuspidatus (Schmeil, 1893) was identified by Anton Brancelj.

The presence of fish in the Scottish lakes was determined from historical or anecdotal sources (Kernan et al. 2009a). Because these data may not be reliable (Kernan et al. 2009c) and only three lakes were fishless, no comparison of Scottish lakes with and without fish was possible. The three fishless lakes had not unique composition of zooplankton. In the Alps and the Tatra Mts., species composition of the fish populations was known at the sampling date (Hořická et al. 2006; Tolotti et al. 2006). Vulnerability to fish predation is dependent on fish density (Knapp et al. 2005), but the density of fish population was not known.

Only a qualitative zooplankton sample was available for SC0140 Gorm Lochan, and only a quantitative zooplankton sample for TY0049 Mittlerer Seewisee.

Data on TOC were available only for the Scottish lakes and data on DOC only for the continental lakes. The median DOC/TOC ratio in oligotrophic lakes is 0.9 (Wetzel 2001). In alpine and mountain forest lakes in Europe, DOC/TOC ranges from 0.69 to 0.92 with Pearson correlation coefficients $r^{2}$ being from 0.53 to 0.98 (Camarero et al. 1999; Winder et al. 2001; Rosén 2005; Nedbalová et al. 2006). In this paper, DOC and TOC were thus regarded as a single variable and named DTOC (dissolved or total organic carbon).

Multiple regression analysis and principal component analysis (PCA) of data were performed using PAST software version 1.94b (the description of PAST give Hammer et al. 2001). Linear regression was used to find a linear relationship between dependent variable $y$ and explanatory variables $x_{n}\left(y=a+b_{1} x_{1}+b_{2} x_{2}+\ldots\right)$. The values of the dependent variable $y$ were assigned to zooplankton species or groups of species according to their expected preference for different size categories of food. For example, value 1 was assigned to Diaphanosoma brachyurum with fine filter meshes, value 0 to Daphnia rosea with intermediate filter meshes and value -1 to Holopedium gibberum with coarse filter meshes. If two species were present in one lake, both values were averaged. Principal component analysis is a procedure for finding hypothetical variables (components) which account for as much of the variance in multidimensional data as possible (Hammer et al. 2001). These new variables are linear combinations of the original variables. PCA results were displayed in a correlation biplot (information on correlation biplots give Legendre \& Legendre 1998).

\section{Results}

A list of all surveyed lakes with their geographical coordinates, area, depth, altitude, crustacean taxa found in zooplankton, $\mathrm{pH}$, dissolved or total organic carbon concentration (DTOC), chlorophyll-a (chl-a) concentration, and fish presence is shown in Table 1. Mean characteristics of lakes grouped in the three lake districts are compared in Table 2.

Tab 2: Comparison of characteristics of lakes in three areas (lake districts): Scotland (code SC), the Alps (code TY), the Tatra Mts. (code TA). TN - total nitrogen, TP - total phosphorus, DTOC - dissolved or total organic carbon, chl-a - chlorophyll-a concentration, bacteria - total bacteria and filamentous biomass, bare ground bare ground (without vegetation) in catchment, ice cover - ice cover duration

\begin{tabular}{|c|c|c|c|c|c|c|c|c|c|c|c|c|}
\hline \begin{tabular}{|l|} 
Code \\
\end{tabular} & & \begin{tabular}{|l|} 
Latitude \\
\end{tabular} & \begin{tabular}{|l|} 
Longitude \\
\end{tabular} & \begin{tabular}{|l|} 
Altitude \\
\end{tabular} & pH & $\mathbf{C l}$ & TP & DTOC & Chl-a & Bacteria & \begin{tabular}{|l|} 
Bare ground \\
\end{tabular} & \begin{tabular}{|l|} 
Ice cover \\
\end{tabular} \\
\hline & & & & $\mathrm{m}$ & & $\mathrm{mg} \mathrm{dm}^{-3}$ & $\mu g \mathrm{dm}^{-3}$ & $\mathrm{mg} \mathrm{C} \mathrm{dm}{ }^{-3}$ & $\mu \mathrm{gdm}^{-3}$ & $\mu \mathrm{g} \mathrm{C} \mathrm{dm}^{-3}$ & $\%$ & days \\
\hline SC & mean & 57,33 & $-4,88$ & 679 & 6,31 & 5,1 & 2,8 & 2,83 & 0,64 & 21,33 & 32,9 & 127 \\
\hline SC & minimum & 56,56 & $-6,22$ & 520 & 5,42 & 1,67 & 2,5 & 0,7 & 0,14 & 1,97 & 0,5 & 113 \\
\hline SC & median & 57,26 & $-4,95$ & 680 & 6,19 & 4,65 & 2,5 & 2,4 & 0,44 & 20,61 & 22,8 & 127 \\
\hline SC & maximum & 58,42 & $-3,23$ & 920 & 7,14 & 9,72 & 6 & 9,2 & 2,04 & 45,67 & 81,9 & 140 \\
\hline $\mathrm{TA}$ & mean & 49,19 & 20,05 & 1843 & 6,5 & 0,17 & 2,6 & 0,54 & 1,37 & 17,04 & 62,4 & 198 \\
\hline $\mathrm{TA}$ & minimum & 49,14 & 19,63 & 1395 & 4,83 & 0,11 & 0,7 & 0,05 & 0,03 & 0,47 & 0 & 168 \\
\hline TA & median & 49,19 & 20,04 & 1870 & 6,62 & 0,17 & 2,1 & 0,32 & 0,84 & 12,05 & 65 & 199 \\
\hline TA & maximum & 49,23 & 20,23 & 2145 & 7,28 & 0,23 & 14,7 & 5,15 & 11,17 & 169,82 & 85 & 223 \\
\hline TY & mean & 47,11 & 11,55 & 2376 & 7,31 & 0,12 & 3,7 & 0,71 & 2,23 & 19,23 & 47 & 212 \\
\hline $\mathrm{TY}$ & minimum & 46,8 & 10,48 & 1840 & 5,66 & 0,07 & 1,6 & 0,25 & 0,21 & 3,48 & 3 & 179 \\
\hline TY & median & 47,19 & 11,41 & 2429 & 7,21 & 0,1 & 2,6 & 0,67 & 1,11 & 19,07 & 47,8 & 216 \\
\hline TY & maximum & 47,43 & 12,77 & 2796 & 8,82 & 0,25 & 8,5 & 1,64 & 18,65 & 30,49 & 95 & 231 \\
\hline
\end{tabular}


Tab 3: The results of linear regression analyses fitting lines $\left(\mathrm{y}=\mathrm{a}+\mathrm{b}_{1} \mathrm{x}_{1}+\mathrm{b}_{2} \mathrm{x}_{2} \ldots\right)$ to data. The values assigned to zooplankton groups were used as dependent variable (y) and $\mathrm{pH}$, depth $(\mathrm{m})$, chlorophyll-a $\left(\mu \mathrm{g} \mathrm{dm}^{-3}\right)$ and DTOC (mg C dm${ }^{-3}$ ) were explaining variables $(x)$. P values of $b_{i}$ coefficients are the results of t-tests. $R^{2}$ is the coefficient of determination. Area codes: SC - Scotland, TY - the Alps, TA - the Tatra Mts. In the Tatra Mts. and the Alps, only zooplankton occurrences in quantitative samples with abundances higher than $0.05 \mathrm{dm}^{-3}$ were included. If only lakes without fish are included in an analysis, it is stated in the Group column.

\begin{tabular}{|c|c|c|c|c|c|c|c|c|c|}
\hline \begin{tabular}{|c|} 
Analysis \\
Nr.
\end{tabular} & $\begin{array}{l}\text { Area } \\
\text { code }\end{array}$ & Group & \begin{tabular}{|c|}
$\begin{array}{c}\text { Dependent variable } \\
\text { value }(y)\end{array}$ \\
\end{tabular} & $\mathbf{N}$ & \begin{tabular}{|l|} 
Explanatory \\
variables (x)
\end{tabular} & Slope (b) & \begin{tabular}{|l|} 
P value \\
(slope)
\end{tabular} & $\mathbf{R}^{2}$ & $\begin{array}{c}\text { P value } \\
\text { (overall) }\end{array}$ \\
\hline \multirow[t]{3}{*}{1} & $1 \mathrm{SC}$ & Diaphanosoma brachyurum & 1 & 23 & DTOC & 0,15 & 0,005 & 0,32 & 0,005 \\
\hline & & Daphnia rosea & 0 & & & & & & \\
\hline & & Holopedium gibberum & -1 & & & & & & \\
\hline \multirow[t]{3}{*}{2} & $2 \mathrm{SC}$ & Conochilus hippocrepis & 1 & 18 & DTOC & $-0,21$ & 0,044 & 0,23 & 0,044 \\
\hline & & Conochilus unicornis & -1 & & & & & & \\
\hline & 3 SC & $\begin{array}{l}\text { Pelagic Rotifera: Ascomorpha ovalis, Asplanchna priodonta, Collotheca } \\
\text { mutabilis, Conochilus hippocrepis, Conochilus unicornis, Gastropus } \\
\text { stylifer, Kellicottia longispina, Keratella cochlearis, Keratella quadrata, } \\
\text { Ploesoma hudsoni, Polyarthra remata, Polyarthra vulgaris and } \\
\text { Synchaeta sp. }\end{array}$ & $\begin{array}{l}\text { Number of species in } \\
\text { one lake }\end{array}$ & 29 & DTOC & 0,58 & 0,025 & 0,17 & 0,025 \\
\hline & 4 SC & $\begin{array}{l}\text { Pelagic Rotifera: Ascomorpha ovalis, Asplanchna priodonta, Collotheca } \\
\text { mutabilis, Conochilus hippocrepis, Conochilus unicornis, Gastropus } \\
\text { stylifer, Kellicottia longispina, Keratella cochlearis, Keratella quadrata, } \\
\text { Ploesoma hudsoni, Polyarthra remata, Polyarthra vulgaris and } \\
\text { Synchaeta sp. }\end{array}$ & $\begin{array}{l}\text { Number of species in } \\
\text { one lake }\end{array}$ & 29 & DTOC & 0,65 & 0,013 & 0,38 & 0,006 \\
\hline & & & & & $\mathrm{pH}$ & 2,44 & 0,058 & & \\
\hline & & & & & $\log _{10}$ (depth) & 3,36 & 0,018 & & \\
\hline \multirow[t]{2}{*}{5} & 5 SC & Keratella cochlearis, Polyarthra remata & 1 & 26 & DTOC & 0,08 & 0,038 & 0,17 & 0,038 \\
\hline & & Keratella hiemalis, Polyarthra dolichoptera & -1 & & & & & & \\
\hline \multirow[t]{2}{*}{6} & \begin{tabular}{|l|l|} 
TATY \\
\end{tabular} & Keratella cochlearis, Polyarthra remata & 1 & 38 & DTOC & 0,19 & 0,009 & 0,18 & 0,009 \\
\hline & & Keratella hiemalis, Polyarthra dolichoptera & -1 & & & & & & \\
\hline \multirow[t]{3}{*}{7} & \begin{tabular}{|l|l|} 
TATY \\
\end{tabular} & Daphnia rosea & 1 & 19 & $\mathrm{pH}$ & 0,58 & 0,012 & 0,56 & 0,002 \\
\hline & & Daphnia pulicaria & 0 & & $\log _{10}$ (depth) & $-1,02$ & 0,003 & & \\
\hline & & Holopedium gibberum & -1 & & & & & & \\
\hline \multirow[t]{3}{*}{8} & \begin{tabular}{|l|l|} 
TATY \\
\end{tabular} & Daphnia rosea & 1 & 19 & DTOC & 1,25 & 0,037 & 0,23 & 0,037 \\
\hline & & Daphnia pulicaria & 0 & & & & & & \\
\hline & & Holopedium gibberum & -1 & & & & & & \\
\hline \multirow[t]{3}{*}{9} & 9 TATY & Daphnia rosea & 1 & 19 & chlorophyll-a & $-0,85$ & 0,077 & 0,17 & 0,077 \\
\hline & & Daphnia pulicaria & 0 & & & & & & \\
\hline & & Holopedium gibberum & -1 & & & & & & \\
\hline \multirow[t]{3}{*}{10} & 0 TATY & Daphnia rosea, no fish & 1 & 13 & $\mathrm{pH}$ & 0,92 & 0,011 & 0,61 & 0,009 \\
\hline & & Daphnia pulicaria, no fish & 0 & & $\log _{10}$ (depth) & $-1,14$ & 0,008 & & \\
\hline & & Holopedium gibberum, no fish & -1 & & & & & & \\
\hline \multirow[t]{3}{*}{11} & 1 TATY & Daphnia rosea, no fish & 1 & 13 & DTOC & 2,72 & 0,027 & 0,37 & 0,027 \\
\hline & & Daphnia pulicaria, no fish & 0 & & & & & & \\
\hline & & Holopedium gibberum, no fish & -1 & & & & & & \\
\hline \multirow[t]{2}{*}{12} & 2 TATY & Arctodiaptomus alpinus & 1 & 44 & chlorophyll-a & $-0,13$ & 0,038 & 0,1 & 0,038 \\
\hline & & Cyclops abyssorum, Acanthocyclops vernalis & -1 & & & & & & \\
\hline \multirow[t]{2}{*}{13} & \begin{tabular}{|l|l|} 
TATY \\
\end{tabular} & Arctodiaptomus alpinus & 1 & 44 & $\mathrm{pH}$ & 0,43 & 0,006 & 0,31 & 0,001 \\
\hline & & Cyclops abyssorum, Acanthocyclops vernalis & -1 & & $\log _{10}$ (depth) & $-0,92$ & 0,003 & & \\
\hline \multirow[t]{2}{*}{14} & 4 TATY & Arctodiaptomus alpinus, no fish & 1 & 26 & $\mathrm{pH}$ & 0,53 & 0,007 & 0,45 & 0,001 \\
\hline & & Cyclops abyssorum, Acanthocyclops vernalis, no fish & -1 & & $\log _{10}$ (depth) & $-1,3$ & 0,002 & & \\
\hline \multirow[t]{2}{*}{15} & 5 TATY & Arctodiaptomus alpinus, no fish & 1 & 26 & DTOC & 0,82 & 0,056 & 0,24 & 0,041 \\
\hline & & Cyclops abyssorum, Acanthocyclops vernalis, no fish & -1 & & chlorophyll-a & $-0,47$ & 0,016 & & \\
\hline \multirow[t]{2}{*}{16} & 6 TATY & Cyclops abyssorum, no fish & 1 & 17 & $\mathrm{pH}$ & 0,78 & $<0.001$ & 0,87 & $<0.001$ \\
\hline & & Acanthocyclops vernalis, no fish & -1 & & $\log _{10}$ (depth) & 0,55 & 0,041 & & \\
\hline \multirow[t]{2}{*}{17} & \begin{tabular}{l|l} 
TATY \\
\end{tabular} & Cyclops abyssorum, no fish & 1 & 17 & DTOC & $-0,45$ & 0,003 & 0,46 & 0,003 \\
\hline & & Acanthocyclops vernalis, no fish & -1 & & & & & & \\
\hline 18 & \begin{tabular}{|l|l|} 
B TATY \\
\end{tabular} & Cyclops abyssorum, no fish & 1 & 17 & chlorophyll-a & $-0,22$ & $<0.001$ & 0,57 & $<0.001$ \\
\hline & & Acanthocyclops vernalis, no fish & -1 & & & & & & \\
\hline 19 & $9 \mathrm{SC}$ & $\begin{array}{l}\text { Arctodiaptomus laticeps, Eudiaptomus gracilis, Mixodiaptomus } \\
\text { laciniatus }\end{array}$ & 1 & 13 & $\log _{10}(\mathrm{DTOC})$ & 2,81 & 0,029 & 0,36 & 0,029 \\
\hline & & Cyclops abyssorum, Megacyclops viridis & -1 & & & & & & \\
\hline & & (lakes with both groups are not included) & & & & & & & \\
\hline 20 & 0 & Arctodiaptomus laticeps & 1 & 21 & chlorophyll-a & $-0,47$ & 0,037 & 0,46 & 0,012 \\
\hline & & Eudiaptomus gracilis & 0 & & $\mathrm{pH}$ & 0,82 & 0,012 & & \\
\hline & & Mixodiaptomus laciniatus & -1 & & $\log _{10}$ (depth) & 0,89 & 0,015 & & \\
\hline 21 & 1 TATY & Daphnia galeata, D. rosea, $D$. pulicaria & 1 & 23 & DTOC & $-1,21$ & 0,005 & 0,32 & 0,005 \\
\hline & & Bosmina longirostris & -1 & & & & & & \\
\hline 22 & \begin{tabular}{|l|l|} 
TATY \\
\end{tabular} & Daphnia galeata, $D$. rosea, $D$. pulicaria & 1 & 23 & chlorophyll-a & $-0,35$ & 0,001 & 0,4 & 0,001 \\
\hline & & Bosmina longirostris & -1 & & & & & & \\
\hline 23 & 3 TATY & fish present & 1 & 58 & $\begin{array}{l}\log _{10}(\text { chlorop } \\
\text { hyll-a) }\end{array}$ & 0,72 & 0,042 & 0,07 & 0,042 \\
\hline & & fish absent & -1 & & & & & & \\
\hline 24 & \begin{tabular}{|l|l}
4 & TATY \\
\end{tabular} & fish present & 1 & 58 & $\log _{10}$ (DTOC) & 1,16 & 0,002 & 0,16 & 0,002 \\
\hline & & fish absent & -1 & & & & & & \\
\hline
\end{tabular}


The relationship of zooplankton species groups to the biotic variables DTOC and chlorophyll-a and the abiotic variables $\mathrm{pH}$ and depth inferred from regression or multiple regression analyses is shown in Table 3. Regression analysis is used to find out whether the variable y describing in most cases the food size preference of zooplankton species is dependent on various environmental characteristics $\left(\mathrm{x}_{\mathrm{i}}\right)$. Nearly all slope coefficients $\left(\mathrm{b}_{\mathrm{i}}\right)$ from regressions are statistically significant ( $\mathrm{P}$ value $<0.05)$. If a slope coefficient is positive, then the dependent variable y increases with the increase of this explaining variable. If a slope coefficient is negative, then the dependent variable y increases with the decrease of this explaining variable. In other words, species represented by high values of y prefer high values of environmental characteristics with positive slope coefficients and low values of environmental characteristics with negative slope coefficients.

DTOC is the key variable in Scotland. As to cladocerans, Diaphanosoma brachyurum prefers high DTOC, Daphnia rosea intermediate, and Holopedium gibberum low values (Table 3, analysis 1). In the Tatra Mts. and the Alps, Daphnia rosea prefers high DTOC (Table 3, analysis 8) and low chlorophyll-a (Table 3, analysis 9), Daphnia pulicaria intermediate, and Holopedium gibberum low DTOC and high chlorophyll-a. In Scotland, the calanoid copepods Arctodiaptomus laticeps, Eudiaptomus gracilis, and Mixodiaptomus laciniatus prefer higher DTOC than the cyclopoid copepods Cyclops abyssorum and Megacyclops viridis (Table 3, analysis 19). This relationship is significant if only lakes with either cyclopoid copepods or calanoid copepods are included, while lakes with both groups not. In the fishless lakes of the Tatra Mts. and the Alps, the calanoid copepod Arctodiaptomus alpinus prefers higher DTOC and lower chlorophyll-a (Table 3, analysis 15) than the cyclopoid copepods Cyclops abyssorum and Acanthocyclops vernalis. Both in the Scottish lakes (Table 3, analysis 5) and the lakes of the Tatra Mts. and the Alps (Table 3, analysis 6), the rotifers Keratella cochlearis and Polyarthra remata prefer higher DTOC than Keratella hiemalis and Polyarthra dolichoptera. In Scotland, the rotifer Conochilus unicornis prefers higher DTOC than C. hippocrepis (Table 3, analysis 2). In Scotland, the number of the pelagic rotifer taxa Ascomorpha ovalis, Asplanchna priodonta, Collotheca mutabilis, Conochilus hippocrepis, Conochilus unicornis, Gastropus stylifer, Kellicottia longispina, Keratella cochlearis, Keratella quadrata, Ploesoma hudsoni, Polyarthra remata, Polyarthra vulgaris and Synchaeta sp. present in one lake increases with increasing DTOC (Table 3, analysis 3).

In the Tatra Mts. and the Alps, zooplankton species groups preferring different DTOC values prefer usually different $\mathrm{pH}$ and depth values, with $\mathrm{pH}$ and depth negatively correlated. Daphnia rosea prefers high $\mathrm{pH}$ and small depth, Daphnia pulicaria intermediate, and Holopedium gibberum low pH and big depth (Table 3, analyses 7 and 10). In the lakes of the Tatra Mts. and the Alps, the calanoid copepod Arctodiaptomus alpinus prefers higher $\mathrm{pH}$ and smaller depth than the cyclopoid copepods Cyclops abyssorum and Acanthocyclops vernalis (Table 3, analyses 13 and 14). In PCA analysis of all lakes, the direction of increasing depth and chlorophyll-a, and decreasing $\mathrm{pH}$ and DTOC approximately agrees with the axis 2 (Fig. 2).

Some other species are also dependent on $\mathrm{pH}$ and depth, but $\mathrm{pH}$ and depth are positively correlated. As regards calanoid copepods in Scotland, Arctodiaptomus laticeps prefers high $\mathrm{pH}$ and depth and low chlorophyll-a, Eudiaptomus gracilis intermediate, and Mixodiaptomus laciniatus low $\mathrm{pH}$ and depth and high chlorophyll-a (Table 3, analysis 20). As regards cyclopoid copepods in the Tatra Mts. and the Alps, Cyclops abyssorum prefers higher $\mathrm{pH}$ and depth and lower chlorophyll-a and DTOC than Acanthocyclops vernalis in fishless lakes (Table 3, analyses 16, 17 and 18). In PCA analysis of all lakes, the direction of pH and depth both decreasing and DTOC and chlorophyll-a both increasing approximately agrees with the axis 1 (Fig. 2). 
In the Tatra Mts. and the Alps, fish are found in lakes with high DTOC and chlorophyll-a (Table 3, analyses 23 and 24). Species often tolerating fish presence are Bosmina longirostris, Daphnia rosea, and Cyclops abyssorum, whereas Arctodiaptomus alpinus and Daphnia pulicaria are usually found in fishless lakes (Table 4). In the Tatra Mts. and the Alps, the cladoceran Bosmina longirostris prefers higher chlorophyll-a and DTOC than the cladocerans Daphnia galeata, D. rosea and D. pulicaria (Table 3, analyses 21 and 22). Species tolerating fish are species occurring at high DTOC and chlorophyll-a: C. abyssorum occurs mostly at high chlorophyll-a (Table 3, analysis 12), B. longirostris at high both DTOC and chlorophylla (Table 3, analyses 21 and 22), and D. rosea at high DTOC (Table 3, analyses 8 and 11).

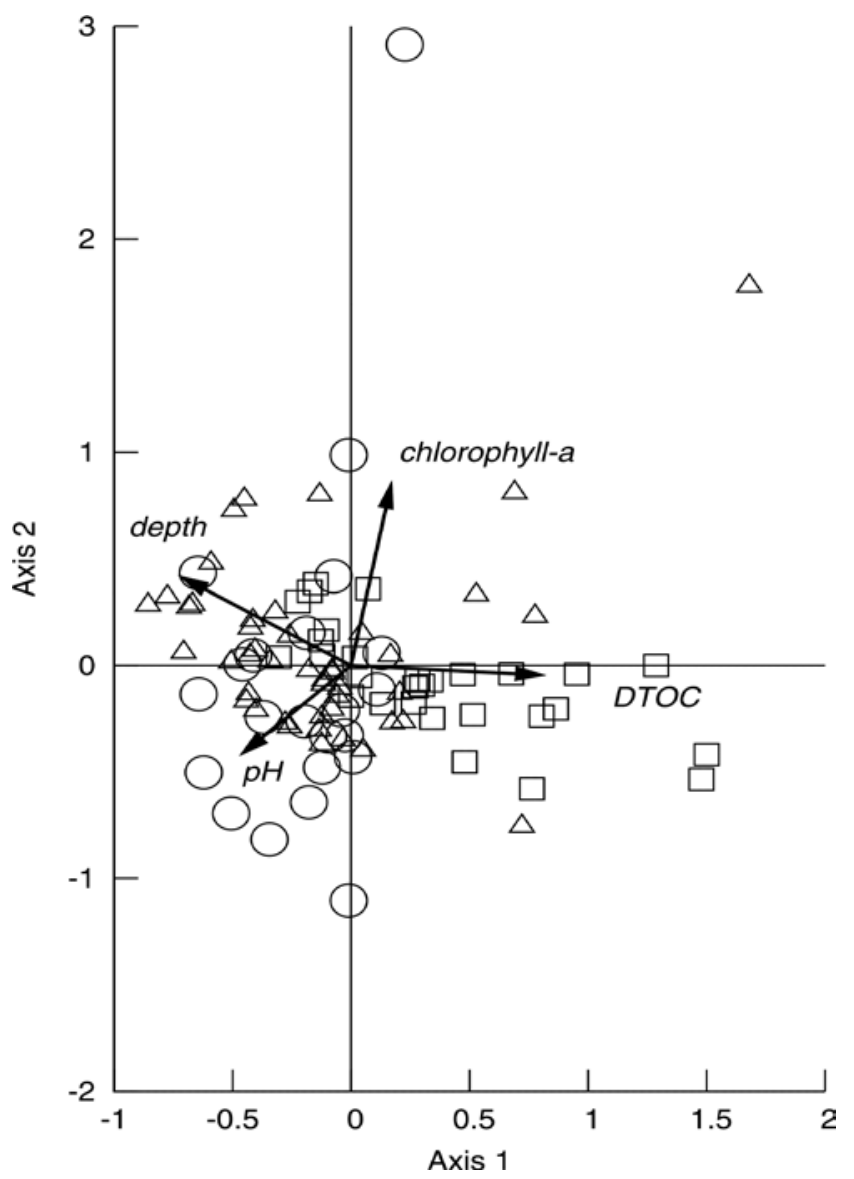

Fig. 2: Correlation biplot based on PCA of characteristics of 96 lakes in the Tatra Mts. $(\Delta)$, the Alps $(\circ)$ and Scotland ( $\square$ ). Depth was logarithmically transformed ( $\log _{10}$ (depth)). Axes 1, 2, and 3 explained 34\%, 26\%, and $23 \%$ of variance, respectively. The species scores (points) were multiplied by 0.5 for display purposes.

\section{Discussion}

In the lakes in the Tatra Mts. and the Alps, microfiltrators with fine filter meshes are replaced by macrofiltrators with coarse filter meshes in the direction of increasing depth and decreasing $\mathrm{pH}$, or increasing chlorophyll-a and decreasing DTOC (Table 3). Filter mesh size is known to predict retention efficiency for bacteria (Brendelberger 1991). The calanoid copepod Arctodiaptomus alpinus is replaced by the cyclopoid copepods Cyclops abyssorum and Acanthocyclops vernalis (Table 3). Both cyclopoid and calanoid copepods are macrofiltrators with coarse filter meshes and low ability to feed on bacteria (Hessen 1985; Hessen et al. 1989; Hopp \& Maier 2005a). Cyclopoid copepods generally prefer larger, often 
animal, food than calanoid copepods do (Fryer 1957; Einsle 1993). For example, Cyclops spp. have lower clearance rates (Adrian 1991) and ingest less and larger algae (Tóth et al. 1987) in comparison to Eudiaptomus gracilis. Similarly, Daphnia rosea is replaced by D. pulicaria, and D. pulicaria by Holopedium gibberum (Table 3). Daphnia rosea has probably smaller filter mesh size than $D$. pulicaria. The distance of setulae on filter combs is 0.5-0.9 in $D$. longispina (Hessen 1985), whereas it is 0.6-1.8 in D. pulicaria (Geller \& Müller 1981). American $D$. pulicaria was found to have mostly higher ratio of algae clearance rate to bacterial clearance rate than American D. rosea (DeMott \& Kerfoot 1982). H. gibberum has coarse filter meshes and low ability to feed on bacteria (Geller \& Müller 1981; Hessen 1985; Hessen et al. 1989). The change from micro- to macrofiltrators with decreasing $\mathrm{pH}$ and increasing depth may be the consequence of decreasing DTOC and increasing chlorophyll-a concentration. The primary production to bacterioplankton production ratio decreases with increasing allochthonous DOC concentration (Jansson et al. 2000).

Microfitrators are replaced by macrofiltrators in the direction of decreasing DTOC also in Scotland. As to cladocerans, Diaphanosoma brachyurum is replaced by Daphnia rosea, which is replaced by Holopedium gibberum (Table 3). From Diaphanosoma over Daphnia to Holopedium, the filter mesh size increases and the efficiency of feeding on bacteria decreases (Geller \& Müller 1981; Hessen 1985; Hessen et al. 1989). Smaller (Bartoš 1959) rotifers Keratella cochlearis and Polyarthra remata prefer higher DTOC than bigger rotifers Keratella hiemalis and Polyarthra dolichoptera both in Scotland and the lakes of the Tatra Mts. and the Alps (Table 3). Because they are smaller species, they may also prefer smaller prey, and they may possibly be microfiltrators. Similarly, Conochilus unicornis is a smaller species (Bartoš 1959) and prefers higher DTOC than C. hippocrepis in Scotland (Table 3). The rotifers Keratella cochlearis and Conochilus unicornis are known to efficiently ingest bacteria (Bogdan \& Gilbert 1987).

Some species are replaced by other species with $\mathrm{pH}$ and depth both increasing, while DTOC and chlorophyll-a both decreasing (Table 3). Thus, the ratio of chlorophyll-a and DTOC probably little varies in this direction and these species may differ in other properties than in their food size preference. It may be food quality, because reduced light with increasing depth may cause a change from low (high C:P ratio) to high quality (low C:P ratio) food. Increasing intensity of light increases carbon to phosphorus (C:P) ratio of phytoplankton (Hessen et al. 2002; Urabe et al. 2002; Hessen 2008). So, calanoids form a series in the direction of $\mathrm{pH}$ and depth both increasing in Scotland, in order Mixodiaptomus laciniatus, Eudiaptomus gracilis, and Arctodiaptomus laticeps (Table 3). Food quality may increase in this direction. To avoid low quality food, $M$. laciniatus may have other mode of feeding than A. laticeps, selecting only high quality particles. There is also a difference in their life cycles: A. alpinus is scarce in the winter and more abundant in the summer, E. gracilis has both summer and winter generations, and $M$. laciniatus reproduces in the winter, while it is absent in the summer (Einsle 1993). A. alpinus has one population peak during the ice-free period, whereas M. laciniatus is present year round in the alpine Seehornsee Lake in Austria (Luger et al. 2000). In the Tatra Mts., the distribution of other species of the genera Mixodiaptomus and Arctodiaptomus is similar to the distribution of these genera in Scotland. In the Tatra Mts., Mixodiaptomus tatricus (Wierzejski, 1883) lives in small acidic lakes with fluctuating water level in the altitudinal zone of dwarf mountain pine, which are rich in organic matter (Brtek 1977). A. alpinus lives there in the zone of meadows with lower export of organic matter. In the Tatra Mts. and the Alps, the cyclopoid copepod Acanthocyclops vernalis is replaced by the cyclopoid copepod Cyclops abyssorum in the direction of $\mathrm{pH}$ and depth both increasing (Table 3). A. vernalis is smaller than C. abyssorum (Maier 1994; Hopp \& Maier 2005a). In Cyclops abyssorum and C. vicinus, the duration of the postembryonic development increases with decreasing food concentration, whereas smaller species Mesocyclops leuckarti, 
Acanthocyclops robustus, and Thermocyclops crassus are less affected (Hopp \& Maier 2005b). Similarly, they may grow better on low quality food. In Scotland, the number of pelagic rotifer taxa increased in the direction of increasing $\mathrm{pH}$ and depth (Table 3). It may be caused by increasing food quality. Further, the number of the taxa increased with DTOC, which may be caused by increasing bacterioplankton production.

Three species were found to resist fish predation in the Tatra Mts. and the Alps: the cyclopoid copepod Cyclops abyssorum, and the cladocerans Bosmina longirostris and Daphnia rosea (Table 4). C. abyssorum is known to be not seriously preyed upon by fish except egg-carrying females, whose eggs pass viable through fish guts (Gliwicz \& Rowan 1984). B. longirostris and D. rosea are resistant thanks to their small size (Lynch 1979; Knapp et al. 2001; Knapp et al. 2005).

Tab 4: Number of occurrences of the zooplankton species in the Tatra Mts. and the Alps in lakes with and without fish. Only occurrences in quantitative samples with abundances higher than $0.05 \mathrm{dm}^{-3}$ are included.

\begin{tabular}{|l|r|r|}
\hline & fish present & fish absent \\
\hline Bosmina longirostris & 5 & 0 \\
\hline Daphnia galeata & 1 & 1 \\
\hline Daphnia pulicaria & 1 & 8 \\
\hline Daphnia rosea & 5 & 3 \\
\hline Holopedium gibberum & 1 & 1 \\
\hline Acanthocyclops vernalis & 1 & 3 \\
\hline Cyclops abyssorum & 16 & 14 \\
\hline Arctodiaptomus alpinus & 3 & 12 \\
\hline
\end{tabular}

Concluding, I have shown that the zooplankton composition is determined by four variables $\mathrm{pH}$, depth, chlorophyll-a and DTOC. Because DTOC and chlorophyll-a concentrations often correlate with the abiotic variables $\mathrm{pH}$ and depth, DTOC and chlorophyll-a may sometimes be omitted and zooplankton composition may be successfully predicted using only $\mathrm{pH}$ and depth. However, introduction of fish induces changes of zooplankton composition together with an increase of chlorophyll-a and DTOC concentrations. These changes are not dependent on abiotic variables and the prediction of zooplankton composition using only $\mathrm{pH}$ and depth fails.

Acknowledgements: I would like to thank Prof. E. Stuchlík from Charles University in Prague for providing data and zooplankton samples from the EMERGE project. I thank Dr. J. Fott from Charles University in Prague for his help with the identification of zooplankton species. The EMERGE project was funded by the European Union (contract EVK1-CT-1999-00032).

\section{References}

Adrian R. (1991): Filtering and feeding rates of cyclopoid copepods feeding on phytoplankton. Hydrobiologia 210: 217-223.

Andersen T. \& Hessen D.O. (1991): Carbon, nitrogen, and phosphorus content of freshwater zooplankton. Limnol. Oceanogr. 36: 807-814.

Bartoš E. (1959): Víŕníci - Rotatoria. Fauna ČSR 15. Nakladatelství Československé akademie věd, Praha, 972 pp. (in Czech with Russian and German summary)

Bogdan K.G. \& Gilbert J.J. (1987): Quantitative comparison of food niches in some freshwater zooplankton. A multi-tracer-cell approach. - Oecologia 72: 331-340. 
Brendelberger H. (1991): Filter mesh size of cladocerans predicts retention efficiency for bacteria. - Limnol. Oceanogr. 36: 884-894.

Brtek J. (1977): Anostraca, Notostraca, Conchostraca a Calanoida Slovenska II. čast'. Anostraca, Notostraca, Conchostraca a Calanoida der Slowakei II. Teil. - Acta rerum naturalium Musei Nationalis Slovaci 23: 117150. (in Slovak with German summary)

Camarero L., Felip M., Ventura M., Bartumeus F. \& Catalan J. (1999): The relative importance of the planktonic food web in the carbon cycle of an oligotrophic mountain lake in a poorly vegetated catchment (Redó, Pyrenees). - J. Limnol. 58: 203-212.

Camarero L., Rogora M., Mosello R., Anderson N.J., Barbieri A., Botev I., Kernan M., Kopáček J., Korhola A., Lotter A.F., Muri G., Postolache C., Stuchlík E., Thies H. \& Wright R.F. (2009): Regionalisation of chemical variability in European mountain lakes. - Freshw. Biol. 54: 2452-2469.

Catalan J., Ventura M., Brancelj A., Granados I., Thies H., Nickus U., Korhola A., Lotter A.F., Barbieri A., Stuchlík E., Lien L., Bitušík P., Buchaca T., Camarero L., Goudsmit G.H., Kopáček J., Lemcke G., Livingstone D.M., Müller B., Rautio M., Šiško M., Sorvari S., Šporka F., Strunecký O. \& Toro M. (2002): Seasonal ecosystem variability in remote mountain lakes: implications for detecting climatic signals in sediment records. - J. Paleolimnol. 28: 25-46.

Cole J.J., Findlay S. \& Pace M.L. (1988): Bacterial production in fresh and saltwater ecosystems: a crosssystem overview. - Mar. Ecol. - Prog. Ser. 43: 1-10.

DeMott W.R. \& Kerfoot W.C. (1982): Competition among cladocerans: nature of the interaction between Bosmina and Daphnia. - Ecology 63: 1949-1966.

DeMott W.R., Gulati R.D. \& Siewertsen K. (1998): Effects of phosphorus-deficient diets on the carbon and phosphorus balance of Daphnia magna. - Limnol. Oceanogr. 43: 1147-1161.

De Smet W.H. (1997): The Dicranophoridae (Monogononta). In: Rotifera, Vol. 5, The Dicranophoridae (Monogononta) and the Ituridae (Monogononta), Guides to the Identification of the Microinvertebrates of the Continental Waters of the World 12, SPB Academic Publishing bv, The Hague, 1-325.

Einsle U. (1993): Crustacea. Copepoda. Calanoida und Cyclopoida. Süßwasserfauna von Mitteleuropa, Band 8/4-1. Gustav Fischer Verlag, Stuttgart, Jena, New York, 209 pp.

Elser J.J., Hayakawa K. \& Urabe J. (2001): Nutrient limitation reduces food quality for zooplankton: Daphnia response to seston phosphorus enrichment. - Ecology 82: 898-903.

Flössner D. (2000): Die Haplopoda und Cladocera (ohne Bosminidae) Mitteleuropas. Backhuys Publishers, Leiden, 428 pp.

Fryer G. (1957): The food of some freshwater cyclopoid copepods and its ecological significance. - J. Anim. Ecol. 26: 263-286.

Geller W. \& Müller H. (1981): The Filtration Apparatus of Cladocera: Filter Mesh-Sizes and their Implications on Food Selectivity. - Oecologia 49: 316-321.

Gliwicz Z.M. \& Rowan M.G. (1984): Survival of Cyclops abyssorum tatricus (Copepoda, Crustacea) in alpine lakes stocked with planktivorous fish. - Limnol. Oceanogr. 29: 1290-1299.

Goldman J.C. \& Graham S.J. (1981): Inorganic Carbon Limitation and Chemical Composition of Two Freshwater Green Microalgae. - Appl. Environ. Microbiol. 41: 60-70.

Hammer Ø., Harper D.A.T. \& Ryan P.D. (2001): PAST: Paleontological Statistics Software Package for Education and Data Analysis. - Palaeontol. Electron., 4: 1-9. $<$ http://palaeo-electronica.org/2001_1/past/issue1_01.htm>, accessed 2 February 2011.

Hein M. (1997): Inorganic carbon limitation of photosynthesis in lake phytoplankton. - Freshw. Biol. 37: 545552.

Hessen D.O. (1985): Filtering structures and particle size selection in coexisting Cladocera. - Oecologia 66: 368-372.

- (2008): Efficiency, energy and stoichiometry in pelagic food webs; reciprocal roles of food quality and food quantity. - Freshw. Rev. 1: 43-57.

Hessen D.O. \& Andersen T. (1990): Bacteria as a source of phosphorus for zooplankton. - Hydrobiologia 206: 217-223.

Hessen D.O., Andersen T. \& Lyche A. (1989): Differential grazing and resource utilization of zooplankton in a humic lake. - Arch. Hydrobiol. 114: 321-347.

Hessen D.O., Færøvig P.J. \& Andersen T. (2002): Light, nutrients, and P:C ratios in algae: grazer performance related to food quality and quantity. - Ecology 83: 1886-1898.

Hessen D.O. \& Lyche A. (1991): Inter- and intraspecific variations in zooplankton element composition. Arch. Hydrobiol. 121: 343-353.

Hopp U. \& Maier G. (2005a): Implication of the feeding limb morphology for herbivorous feeding in some freshwater cyclopoid copepods. - Freshw. Biol. 50: 742-747.

- (2005b): Survival and development of five species of cyclopoid copepods in relation to food supply: experiments with algal food in a flow-through system. - Freshw. Biol. 50: 1454-1463. 
Hořická Z., Stuchlík E., Hudec I., Černý M. \& Fott J. (2006): Acidification and the structure of crustacean zooplankton in mountain lakes: The Tatra Mountains (Slovakia, Poland). - Biologia, Bratislava 61/Suppl. 18: 121-134.

Jansson M., Bergström A.-K., Blomqvist P. \& Drakare S. (2000): Allochthonous Organic Carbon and Phytoplankton/Bacterioplankton Production Relationships in Lakes. - Ecology 81: 3250-3255.

Kernan M., Brancelj A., Clarke G., Lami A., Raddum G., Straškrábová V., Stuchlík E., Velle G. \& Ventura M. (2009a): Environmental and biological characteristics of high altitude lochs in Scotland. Fundam. - Adv. Limnol. 62: 379-417.

Kernan M., Catalan J., Ventura M. \& Curtis C.J. (2009b): A biological survey of high mountain and high latitude lakes across Europe: aims, sampling strategy, methods and main achievements. - Adv. Limnol. 62: 3-16.

Kernan M., Ventura M., Bitušík P., Brancelj A., Clarke G., Velle G., Raddum G.G., Stuchlík E. \& Catalan J. (2009c): Regionalisation of remote European mountain lake ecosystems according to their biota: environmental versus geographical patterns. - Freshw. Biol. 54: 2470-2493.

Kiefer F. (1978): Freilebende Copepoda. In: Die Binnengewässer, Band 26, Das Zooplankton der Binnengewässer, 2. Teil. E. Schweizerbart'sche Verlagsbuchhandlung, Stuttgart, 1-343.

Knapp R.A., Hawkins C.P., Ladau J. \& McClory J.G. (2005): Fauna of Yosemite National Park lakes has low resistance but high resilience to fish introductions. - Ecol. Appl. 15: 835-847.

Knapp R.A., Matthews K.E. \& Sarnelle O. (2001): Resistance and resilience of Alpine lake fauna to fish introductions. - Ecol. Monogr. 71: 401-421.

Koste W. (1978): Rotatoria. Die Rädertiere Mitteleuropas. Ein Bestimmungwerk, begründet von Max Voigt. Überordnung Monogononta. 2nd ed. Gebrüder Borntraeger, Berlin, Stuttgart, I. Textband 673 pp., II. Tafelband 234 plates.

Legendre P. \& Legendre L. (1998): Numerical Ecology. 2nd English ed. Elsevier Science B.V., Amsterdam, 853 pp.

Luger M.S., Schabetsberger R., Jersabek C.D. \& Goldschmid A. (2000): Life cycles, size and reproduction of the two coexisting calanoid copepods Arctodiaptomus alpinus (IMHOF, 1885) and Mixodiaptomus laciniatus (LILLJEBORG, 1889) in a small high-altitude lake. - Arch. Hydrobiol. 148: 161185.

Lynch M. (1979): Predation, competition, and zooplankton community structure: An experimental study. Limnol. Oceanogr. 24: 253-272.

Maier G. (1994): Patterns of life history among cyclopoid copepods of central Europe. - Freshw. Biol 31: 7786.

Nedbalová L., Vrba J., Fott J., Kohout L., Kopáček J., Macek M. \& Soldán T. (2006): Biological recovery of the Bohemian Forest lakes from acidification. - Biologia, Bratislava 61/Suppl. 20: 453-465.

Negrea Ş. (1983): Cladocera. Fauna Republicii Socialiste România 4/12. Editura Academiei Republicii Socialiste România, Bukureşti, 400 pp.

Nygaard G. (1989): Some Observations on the Irradiance and Carbon Fixation in Grane Langsø. - Int. Rev. ges. Hydrobiol. Hydrogr. 74: 293-319.

Panzenböck M. (2007): Effect of solar radiation on photosynthetic extracellular carbon release and its microbial utilization in alpine and Arctic lakes. - Aquat. Microb. Ecol. 48: 155-168.

Petrusek A., Černý M., Mergeay J. \& Schwenk K. (2007): Daphnia in the Tatra Mountain lakes: multiple colonisation and hidden species diversity revealed by molecular markers. - Fundam. Appl. Limnol. Arch. Hydrobiol. 169/4: 279-291.

Reche L., Pugnetti A., Cruz-Pizarro L. \& Carrillo P. (1996): Relationship between bacteria and phytoplankton in a high-mountain lake: Importance of the organic carbon released by pelagic algae for bacterioplankton. - Adv. Limnol 48: 31-38.

Rosén P. (2005): Total organic carbon (TOC) of lake water during the Holocene inferred from lake sediments and near-infrared spectroscopy (NIRS) in eight lakes from northern Sweden. - Biogeochemistry 76: 503-516.

Rozkošný R. (1980): Klíč vodních larev hmyzu. [Identification key to aquatic larvae of insects]. Československá akademie věd, Praha, 524 pp. (in Czech)

Ruttner-Kolisko A. (1972): Rotatoria. In: Die Binnengewässer, Band 26, Das Zooplankton der Binnengewässer, 1. Teil. E. Schweizerbart'sche Verlagsbuchhandlung, Stuttgart, 99-234.

Segers H. (1995): Rotifera. Vol. 2: The Lecanidae (Monogononta). Guides to the Identification of the Invertebrates of the Continental Waters of the World 6. SPB Academic Publishing bv, The Hague, 226 pp.

Šrámek-Hušek R., Straškraba M. \& Brtek J. (1962): Lupenonožci - Branchiopoda. Fauna ČSSR 16. Nakladatelství Československé akademie věd, Praha, 472 pp. (in Czech with Russian and German summary)

Straškrábová V., Bertoni R., Blažo M., Callieri C., Forsström L., Fott J., Kernan M., Macek M., Stuchlík E. \& Tolotti M. (2009): Structure of pelagic microbial assemblages in European mountain lakes during ice-free season. - Adv. Limnol 62: 19-53. 
Straškrábová V. \& Stuchlík E. (2000): EMERGE sampling protocol 7. Plankton food web. $<$ http://www.mountain-lakes.org/emerge/>, accessed 26.9.2012.

The MOLAR Water Chemistry Group (1999): The MOLAR project: atmospheric deposition and lake water chemistry. - J. Limnol. 58: 88-106.

Tolotti M., Manca M., Angeli N., Morabito G., Thaler B., Rott E. \& Stuchlík E. (2006): Phytoplankton and zooplankton associations in a set of Alpine high altitude lakes: geographic distribution and ecology. - Hydrobiologia 562: 99-122.

Tóth L.G., Zánkai N.P. \& Messner O.M. (1987): Alga consumption of four dominant planktonic crustaceans in Lake Balaton (Hungary). - Hydrobiologia 145: 323-332.

Urabe J., Kyle M., Makino W., Yoshida T., Andersen T. \& Elser J.J. (2002): Reduced light increases herbivore production due to stoichiometric effects of light: nutrient balance. - Ecology 83: 619-627.

Vadstein O., Olsen Y., Reinertsen H. \& Jensen A. (1993): The role of planktonic bacteria in phosphorus cycling in lakes - Sink and link. - Limnol. Oceanogr. 38: 1539-1544.

Wathne B.M. \& Hansen H.E. (1997): MOLAR. Measuring and modelling the dynamic response of remote mountain lake ecosystem to environmental change: A program of Mountain lake Research. MOLAR Project Manual. NIVA Report, 0-96061, Oslo.

Wetzel R.G. (2001): Limnology: Lake and River Ecosystems. 3rd ed. Academic Press, San Diego, 1006 pp.

Williams T.G. \& Turpin D.H. (1987): Photosynthetic kinetics determine the outcome of competition for dissolved inorganic carbon by freshwater microalgae: implications for acidified lakes. - Oecologia 73: 307311.

Winder M., Monaghan M.T. \& Spaak P. (2001): Have Human Impacts Changed Alpine Zooplankton Diversity over the Past 100 Years? - Arct. Antarct. Alp. Res. 33: 467-475.

Author's address: Ivan Skála, Povodí Vltavy, s. p. (Vltava River Authority), Na Hutmance 5a, 15800 Praha 5, Czech Republic

E-mail: ivan.skala@pvl.cz 